\title{
1 Experimental and Numerical Study on Vortex-Induced 2 Motions of a Deep-Draft Semi-Submersible
}

3 Yibo Liang a ${ }^{\text {a }}$ Longbin Tao ${ }^{\mathrm{a}, 1}$, Longfei Xiao ${ }^{\mathrm{b}, \mathrm{c}}$, Mingyue Liu ${ }^{\mathrm{b}, \mathrm{c}}$

$4{ }^{a}$ School of Marine Science and Technology, Newcastle University, Newcastle upon Tyne, NE1 7RU, $5 \quad U K$

$6 \quad{ }^{b}$ State Key Laboratory of Ocean Engineering, Shanghai Jiao Tong University, Shanghai, 200240, 7 China

$8{ }^{c}$ Collaborative Innovation Centre for Advanced Ship and Deep-Sea Exploration, Shanghai, 20040, 9 China

10 Abstract

11 An experimental study on Vortex-Induced Motions (VIM) of a Deep-Draft Semi-Submersible (DDS) 12 was carried out in a towing tank, with the aim to investigate the VIM effects on the overall 13 hydrodynamics of the structure. In order to study the fluid physics associated with VIM of the DDS, a comprehensive numerical simulation was conducted to examine the characteristics of vortex shedding processes and their interactions due to multiple cylindrical columns. The experimental measurements were obtained for horizontal plane motions including transverse, in-line and yaw motions as well as drag and lift forces on the structure. Spectral analysis was further carried out based on the recorded force time history. These data were subsequently used to validate the numerical model. Detailed numerical results on the vortex flow characteristics revealed that during the "lock-in", the vortex shedding processes of the upstream columns enhance the vortex shedding processes of the downstream columns leading to the rapid increase of the magnitude of the VIM. In addition to the experimental measurements, for the two uniform flow incidences $\left(0^{\circ}\right.$ and $\left.45^{\circ}\right)$ investigated, comprehensive numerical data of the parametric study on the VIM characteristics at wide range of current strength will also serve as quality benchmarks for future study and provide guidance for practical design.

\footnotetext{
${ }^{1}$ Corresponding author. Tel.: +44 (0) 191208 6670; Fax: +44 (0) 191208 5491; E-mail address: longbin.tao@newcastle.ac.uk
} 


\section{Keywords}

27 Vortex-Induced Motions (VIM); Deep-Draft Semi-Submersible (DDS); Model Test; Computational 28 Fluid Dynamics (CFD)

\section{Nomenclature}

$30 \quad A \quad$ Projected area

$31 \quad A_{x} / L \quad$ Non-dimensional characteristics amplitude of in-line motion

$32 \quad A_{y} / L \quad$ Non-dimensional characteristics amplitude of transverse motion

$33 \quad A_{1 / 3} / L \quad$ Non-dimensional significant values of the transverse peaks

$34 \quad B_{L} \quad$ Platform width

$35 \quad B_{T} \quad$ Platform draft

$36 \quad C \quad$ Structural damping

$37 C_{D} \quad$ Drag force coefficient

$38 \quad C_{L} \quad$ Lift force coefficient

$39 \quad D \quad$ Column projected width

$40 \quad f_{s} \quad$ Vortex shedding frequency

$41 f_{0} \quad$ Natural frequency in clam water

$42 \quad \mathrm{Fr} \quad$ Froude number

$43 F_{D}, F_{x}$ Hydrodynamic drag force acting on the structure

$44 \quad F_{L}, F_{y}$ Hydrodynamic lift force acting on the structure

$45 \quad g \quad$ Acceleration of gravity

$46 \quad H \quad$ Immersed column height above the pontoon

$47 \quad K_{x} \quad$ Linear spring constant in the in-line direction 
$48 \quad K_{y} \quad$ Linear spring constant in the transverse direction

$49 \quad L \quad$ Column width

$50 \quad P \quad$ Pontoon height

$51 \quad$ Re Reynolds number

52 rms Root mean square

$53 \quad S \quad$ Distance between centre columns

$54 \quad$ St $\quad$ Strouhal number

$55 \quad T_{0} \quad$ Natural periods in calmwater

$56 \Delta t \quad$ Numerical simulation time step

$57 \quad U, U_{c} \quad$ Current speed

$58 \quad u_{*} \quad$ Friction velocity at the nearest wall

$59 \quad U r \quad$ Reduced velocity

$60 \rho \quad$ Fresh water density

$61 \Delta \quad$ Displacement

$62 \Delta y_{1} \quad$ First layer thickness

$63 \lambda \quad$ Scale ratio

$64 \theta \quad$ Attack angle; Flow incidence

$65 v \quad$ Kinematic viscosity of the fresh water

$66 \omega \quad$ Vorticity magnitude

$67 x, X \quad$ In-line motion

$68 y, Y \quad$ Transverse motion

$69 y^{+} \quad$ Y plus value 
71

Along with the continuing developments in the field of offshore technology, an increasing number of deep-draft floating structures have been fabricated and installed in different deep-water regions around the world such as the Gulf of Mexico (GoM). Deep-draft floating structures have favourable behaviour in vertical plane motions and therefore are easy to accommodate steel risers. Most of the deep-draft floating structures consist of four vertical cylindrical columns with connecting deck and lower pontoon type members. When a current flows past the columns, a complex issue named VIM can generate strong cyclic dynamic effects on the floaters, especially when the vortex shedding frequency is approaching the natural frequency of the structure leading to the so-called "lock-in" phenomenon. VIM is a cyclic rigid body motion induced by vortex shedding on a large floating structure. It is a common practice to increase the draft of the columns in order to achieve the desired hydrodynamic characteristics in vertical plane motions. However, the increase in columns' draft can also lead to more severe VIM. In this context, both experimental and numerical methods are used to investigate the mechanism of VIM and the effects on overall hydrodynamics of the DDS.

In deep-water developments, a favourable motion response of the floater is critical to the safe operations of top-tensioned facilities, as well as the fatigue life of the mooring system and the risers. In the GoM, due to the strong loop currents, VIMs have been often observed since the Genesis Spar platform commissioned in 1997 [1, 2]. Finn et al. [3] and van Dijk et al. [4] investigated VIM effects on different designs of Spar platform. To reduce the potential problems, spiral strakes attached to the hull were examined as an acceptable design approach in order to minimize the VIM phenomenon. Several experiments on Spar VIM were carried out to mitigate VIM, such as Irani and Finn [5], Halkyard et al. [6], Wang et al. [7] and Wang et al. [8]. In the last decade, Computational Fluid Dynamics (CFD) provided a reasonable alternative way to predict VIM on Spar platforms. Halkyard et al. [9], Oakley and Constantinides [10] combined the results from experimental and numerical studies in order to compare the VIM effects on Spar from experimental measurements and CFD predictions. Thiagarajan et al. [11] further investigated a bare cylinder and a cylinder with strakes to study the VIM phenomenon. A guideline of numerical simulation of the VIM on the Spar platform was proposed by Lefevre et al. [12].

The presence of the VIM phenomenon on more complex multiple cylindrical structures, such as Tension-Leg Platforms (TLP) and DDS, is confirmed from field measurements made by Rijken and Leverette [13]. Waals et al. [14] studied the draft effects on VIM. When the draft changed from a typical conventional semi-submersible to a DDS, significant increases of VIM were observed. Hong et al. [15] also reported that deep-draft floaters experience strong VIM. Gonçalves et al. [16] found that even the conventional semi-submersible with appendages can also suffer from VIM. For most of 
Table 1. Summary of the studies on VIM of deep-draft structures (“*” is the numerical result).

\begin{tabular}{|c|c|c|c|c|c|c|c|c|}
\hline & $\lambda$ & $\begin{array}{l}\text { Mass } \\
\text { ratio }\end{array}$ & $H / L$ & $S / L$ & $H / P$ & $\operatorname{Re}$ & $U r$ & $A_{y} / L$ at $45^{\circ}$ \\
\hline $\begin{array}{l}\text { Waals et } \\
\text { al. }[14]\end{array}$ & $1: 70$ & 0.83 & 1.75 & 4.14 & 2.33 & $6 \times 10^{3} \sim 7 \times 10^{4}$ & $4.0 \sim 40.0$ & 0.32 \\
\hline $\begin{array}{l}\text { Rijken and } \\
\text { Leverette }\end{array}$ & $1: 50$ & -- & 2.18 & 3.75 & 4.83 & $\sim 10^{5}$ & $1.0 \sim 15.0$ & 0.48 \\
\hline $\begin{array}{l}\text { Rijken et } \\
\text { al. [21] }\end{array}$ & $1: 48$ & -- & 1.71 & 4.04 & 3.04 & $3 \times 10^{4} \sim 3 \times 10^{5}$ & $5.0 \sim 9.0$ & $0.64[16]$ \\
\hline $\begin{array}{l}\text { Tahar and } \\
\text { Finn [22] }\end{array}$ & $1: 56$ & 0.77 & 1.74 & 3.20 & 4.00 & $\sim 5 \times 10^{5}$ & $2.0 \sim 15.0$ & 0.33 \\
\hline $\begin{array}{l}\text { Lee et al. } \\
{[18]}\end{array}$ & $1: 67$ & -- & 1.78 & 3.50 & 3.62 & $2 \times 10^{4} \sim 9 \times 10^{4}$ & $4.0 \sim 20.0$ & $0.4^{*}$ \\
\hline $\begin{array}{l}\text { Present } \\
\text { study }\end{array}$ & $1: 64$ & 0.91 & 1.90 & 3.72 & 3.70 & $2 \times 10^{4} \sim 1 \times 10^{5}$ & $3.4 \sim 20.2$ & $0.742 / 0.751^{*}$ \\
\hline
\end{tabular}
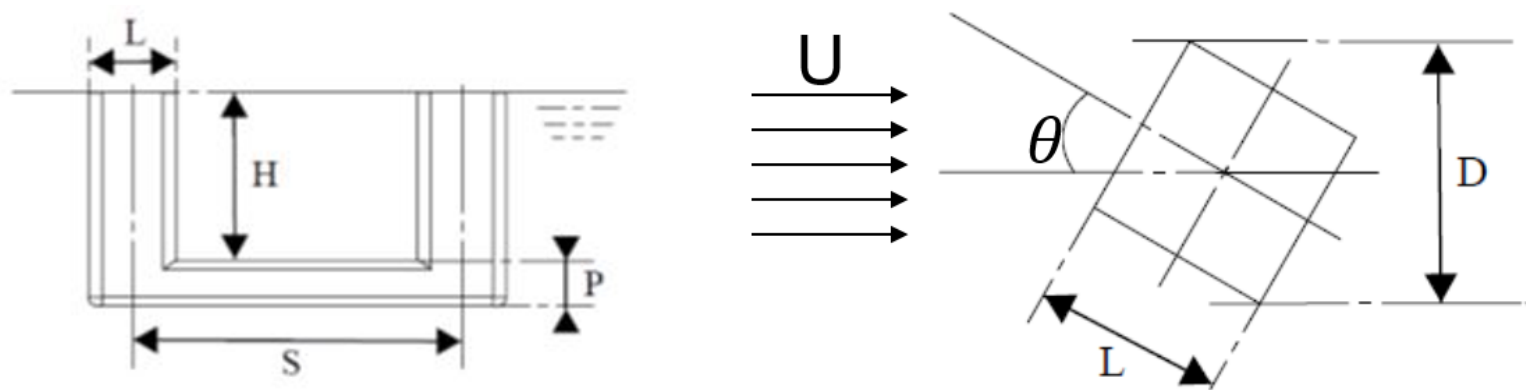

Fig. 1. Characteristic dimensions of a DDS. 


\section{Model test}

\subsection{Model set-up}

121 The experimental set-up is characterized by a DDS model supported above the waterline by four low

122 friction air bearings and a set of equivalent horizontal mooring springs in the Zhejiang Ocean

123 University towing tank with dimension of $130 \times 6 \times 3 \mathrm{~m}$ (length $\times$ width $\times$ depth). The DDS model

124 and experimental set-up in the towing tank are shown in Fig. 2 and Fig. 3.

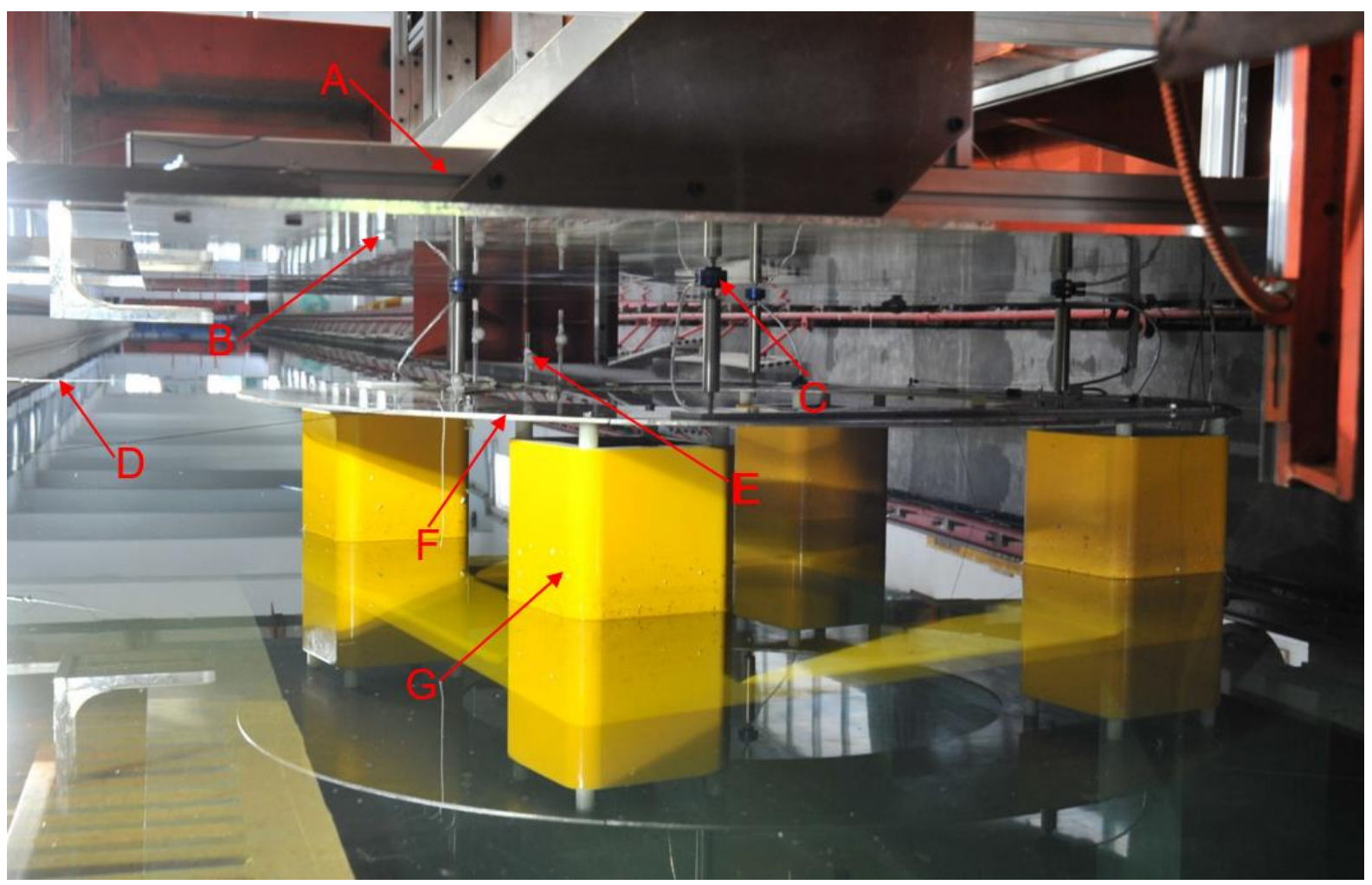
A) Adjustable support structure
B) Smooth horizontal table
C) Low friction air bearing
D) Horizontal spring with load cell
E) Locomotion measure device with 6 degree of freedom
F) Top frame
G) DDS model in scale ratio $\lambda=1: 64$

127 Fig. 2. Experimental set-up in the towing tank. 
Towing direction

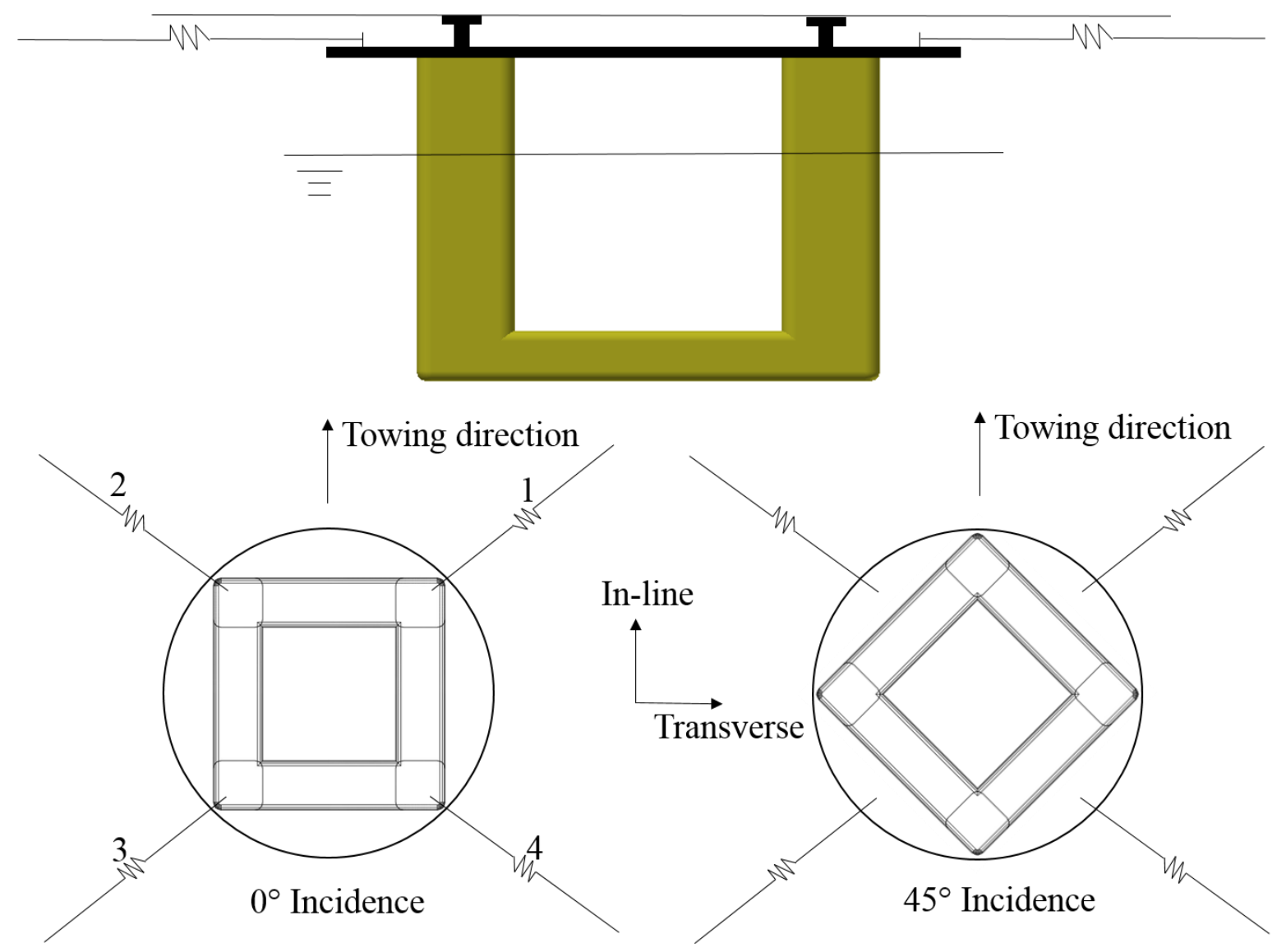

Fig. 3. Schematic of the experimental set-up.

Table 2. Main characteristics of the DDS unit.

\begin{tabular}{lll}
\hline & Prototype $(\mathbf{m})$ & Model $(\mathbf{m})$ \\
\hline Distance between centre columns $(\boldsymbol{S})$ & 72.5 & 1.133 \\
Column width $(\boldsymbol{L})$ & 19.5 & 0.305 \\
Immersed column height above the pontoon $(\boldsymbol{H})$ & 37.0 & 0.578 \\
Pontoon height $(\boldsymbol{P})$ & 10.0 & 0.156 \\
\hline
\end{tabular}

It is important for keeping the similarity between prototype and model. Thus, the Froude scaling approach recommended by van Dijk et al. [23] was used. It is essential to note that the Reynolds number $(\operatorname{Re}=U D / v$, where $U$ is the current velocity, $D$ is the projected width of the column and $v$ is the kinematic viscosity of the fresh water) for the prototype DDS is in the order of $10^{7}$ while the Reynolds number at model scale is significantly lower. Since the DDS model is a relatively bluff body, the flow is expected to separate at the corners of the columns. The vortex shedding phenomenon is mostly independent of the Reynolds number from the transcritical region to the subcritical region. The scale effects for square section shaped structure are less than that for circle 

section shaped structures [2]. The main characteristics of the DDS model are shown in Table 2 with the dimensions defined in Fig. 1.

\subsection{Mooring system}

Four horizontal mooring lines with load cells are attached at the top frame to restrain the horizontal motions of the DDS model. An additional set of four low friction air bearings were developed in order to limit the vertical motions of the DDS model. Only three degrees of freedom motions in horizontal plane (namely transverse, in-line and yaw) were allowed in the test. The horizontal mooring system consists of four horizontal lines with soft springs being employed to provide the horizontal restoring force for the model and match the natural periods in the horizontal plane motions. The mooring lines were set above the water level to avoid disturbing the vortex shedding process. Each mooring line with a load cell was attached to an anchor post on the carriage at one end and to the top deck of the model at the other end. The top deck featuring studs were arranged circumferentially with $15^{\circ}$ spacing interval. When the current incidences need to be changed, the model with the top deck can be rotated accordingly and the horizontal lines are attached to the appropriate studs, allowing the same mooring configuration for the two different current incidence angles. Therefore, the mooring stiffness was kept the same for the two current incidences, aiming to facilitate result comparison [24].

\subsection{Test programme}

In order to investigate the effects of VIM on the DDS model under a reduced velocity ranging from 3.5 to 20.3 , two incidences $\left(0^{\circ}\right.$ and $\left.45^{\circ}\right)$ relative to the towing direction were tested. The definition of motions and towing directions are shown in Fig. 3. A minimum of fifteen oscillation cycles were allowed to occur in order to reflect the quasi-steady state of the experimental VIM phenomenon.

\subsection{Reduced velocity}

The reduced velocity $(U r)$ is defined as:

$U r=\frac{\left(U T_{0}\right)}{D}$

where $U$ is the current speed, $T_{0}$ is the natural period in calm water and $D$ is the projected width of the column.

Table 3. Natural periods of the motions in calm water. 


\begin{tabular}{llll}
\hline Incidences $\left(^{\circ}\right)$ & $\begin{array}{l}\text { Natural period of } \\
\text { transverse motion, } \\
\boldsymbol{T}_{\text {0transverse }}(\mathbf{s})\end{array}$ & $\begin{array}{l}\text { Natural period of in-line } \\
\text { motion, } \\
\boldsymbol{T}_{\text {0in-line }}(\mathbf{s})\end{array}$ & $\begin{array}{l}\text { Natural period of } \\
\text { yaw motion, } \\
\boldsymbol{T}_{\text {oyaw }}(\mathbf{s})\end{array}$ \\
\hline $\mathbf{0}^{\circ}$ & 19.4 & 19.6 & 17.1 \\
$\mathbf{4 5}^{\circ}$ & 20.1 & 19.2 & 18.3 \\
\hline
\end{tabular}

\section{3. Numerical (CFD) simulation}

168

169

170

171

172

\subsection{Computational overview}

To further investigate the fluid physics associated with VIM, a comprehensive numerical study is conducted to examine the vortex shedding characteristics and the vortex dynamics leading to the motions of DDS. A mesh and time step sensitivity assessment has been carried out on the numerical model in order to develop an efficient process followed by the actual VIM simulations.

The detached eddy simulation (DES) was used in this study. For the DES model, the improved delayed detach eddy simulation (IDDES) model [25] with the Spalart-Almaras (SA) [26] was used. Delayed detached eddy simulation (DDES) [27] is a recent modification of detached eddy simulation (DES) [26]. IDDES is a capable model which builds a single set of formulas both for natural (D)DES applications and for wall-modelling in large eddy simulation (WMLES) [25]. In this case, the boundary layers and irrotational regions are solved using SA model. However, when the grid is fine enough, it will emulate a basic large eddy simulation (LES) subgrid scale model in detached flow regions [28]. This approach can improve the boundary layer simulation and in the meantime reduce the computational cost. It is noted that the SA model requires $y^{+}<1$ (where $y^{+}=u * \Delta y_{1} / v$, and where $u_{*}$ denotes the friction velocity at the nearest wall, $\Delta y_{1}$ is the first layer thickness and $v$ is the kinematic viscosity) indicating that the viscous sublayer is properly resolved. All the simulations are carried out using Star-CCM+9.

The main characteristics of the DDS design analysed in this section are given in Table 2. Additionally, a MARIN DDS design [14] was also simulated, aiming to further validate the present numerical model with previously published experiment data. The main characteristics of the MARIN model are shown in Table 4. The scale ratio $\lambda$ for this particular model is 1:70 (the flow velocity is set as $0.17 \mathrm{~m} / \mathrm{s}$ which is the same as in the experiment undertaken by Waals et al. [14]).

Table 4. The main characteristics of the MARIN DDS. 


\begin{tabular}{lll}
\hline & Prototype $(\mathbf{m})$ & Model $(\mathbf{m})$ \\
\hline Distance between centre columns $(\boldsymbol{S})$ & 58.7 & 0.838 \\
Column width $(\boldsymbol{L})$ & 14.0 & 0.200 \\
Immersed column height above the pontoon $(\boldsymbol{H})$ & 35.0 & 0.500 \\
Pontoon height $(\boldsymbol{P})$ & 10.5 & 0.150 \\
\hline
\end{tabular}

191

192

193

194

195

196

197

For all the simulations, the computational domain $9 B_{L} \times 6 B_{L} \times 3 B_{T}$ was used (where $B_{L}$ is the hull width of the DDS and $B_{T}$ is the draft of the DDS, see Fig. 4). It is noted that the computational domains were $6 B_{L} \times 4.5 B_{L} \times 2.8 B_{T}$ and $5 B_{L} \times 4 B_{L} \times 2.2 B_{T}$ in the study by Lee et al. [18]. Tan et al. [17] performed simulation with a domain $27 B_{L} \times 18 B_{L} \times 6.5 B_{T}$ and Liu et al. [29] used a domain of $11 B_{L} \times 6 B_{L} \times 3 B_{T}$. Compared with aforementioned computational domain settings, a $9 B_{L} \times 6 B_{L} \times 3 B_{T}$ domain is considered to be large enough to eliminate the far field effects from the boundaries affecting the flow around the model and the three-dimensional effects from a spanwise cross flow direction.

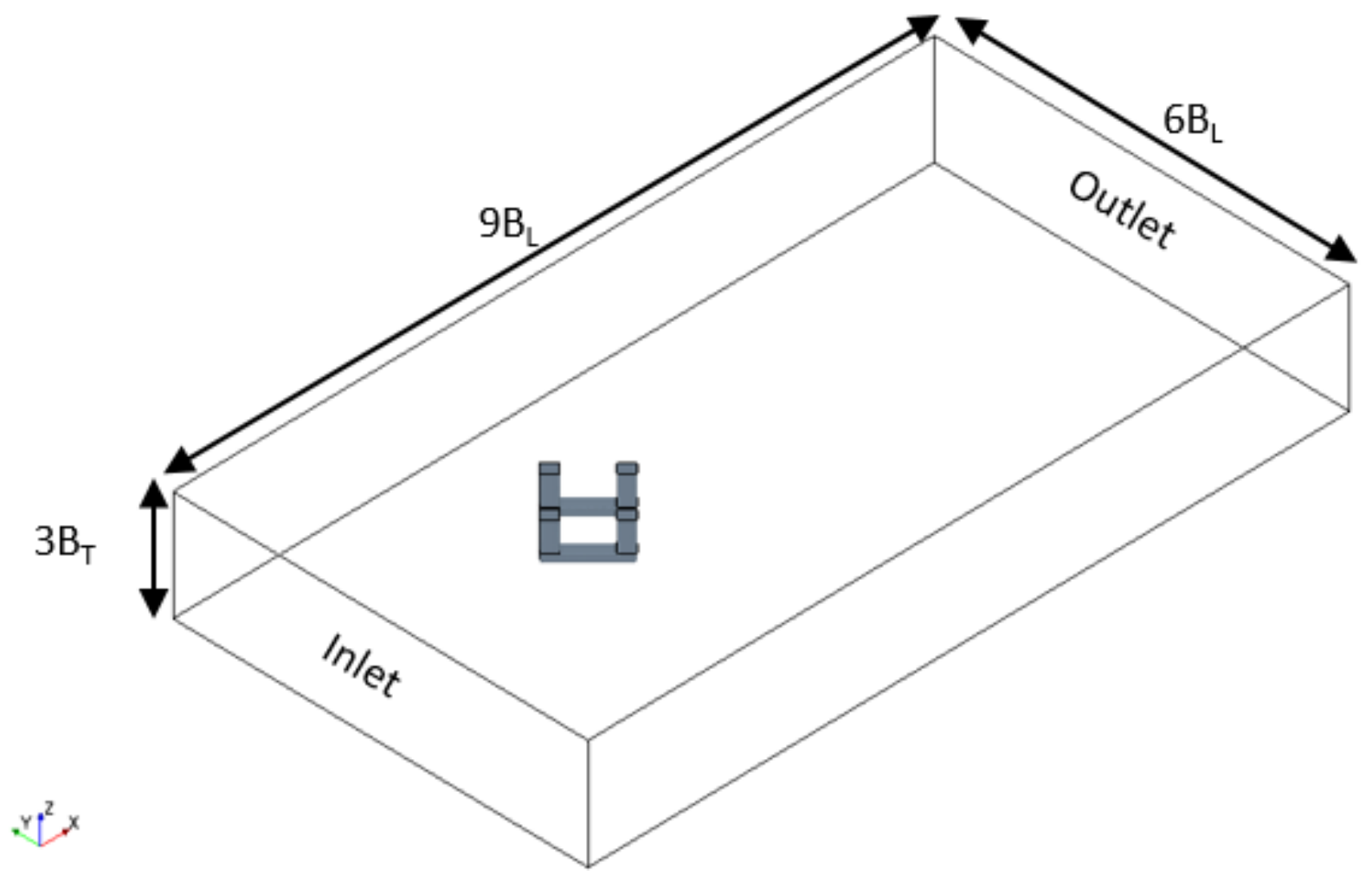

Fig. 4. Computational domain.

The computational domain is modelled with a three-dimensional mesh of elements. A polyhedral mesh [28] is used in this study. The overall element mesh domain is shown at a mid-depth horizontal layer in Fig. 5. In the present study, a near wall refinement method named "Prism Layer Mesher [28]" is adopted. The "Prism Layer Mesher" model is used with a core volume mesh to generate orthogonal 
prismatic cells next to wall surfaces. This layer of cells is necessary to improve the accuracy of the flow solution [28]. The $y^{+}$values are smaller than 1 in all simulations. Another five regional refinements are added in the domain in order to refine both the near wake and the far wake region (see Fig. 5).

The boundary conditions are kept same in all simulations. At the inlet, a uniform and constant velocity of $0.25 \mathrm{~m} / \mathrm{s}$ is specified directly for all sensitivity studies. Along the outlet boundary, the pressure is prescribed to be equal to zero. The velocity at the boundary is extrapolated from the interior using reconstruction gradients [28]. For the body surface of the DDS, a no-slip boundary condition is specified in terms of the tangential velocity which is explicitly set to be zero and the pressure at the boundary is extrapolated from the adjacent cells using reconstruction gradients [28]. It is noted that for the Froude number being quite small $(F r<0.2, F r=U / \sqrt{g D}$, where $U$ is the current velocity, $g$ is the acceleration of gravity and $D$ is the projected width of the column) in all simulations of the present investigation. As observed in the model tests, the free surface effects are rather limited and can be ignored. Therefore, only the submerged geometry is considered, and the geometry of the structure above the waterline will not affect the simulation results. However, the gravity centre, the mass of the structure and the moment of inertia are still using the values from the entire structure design. Thus, the free surface boundary is prescribed as being a symmetry boundary.

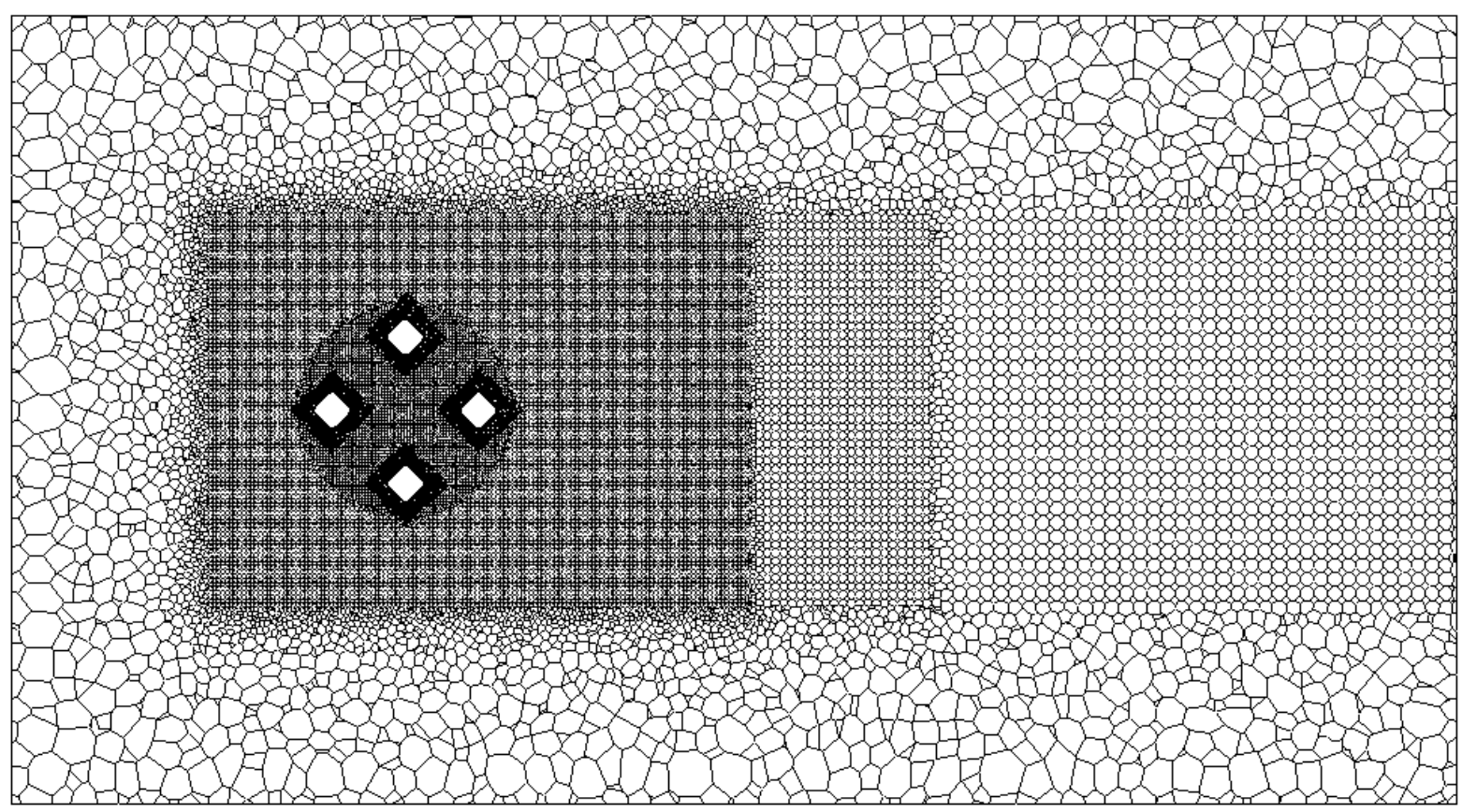

Fig. 5. Visualization of the mesh at the middle draft level of the DDS ( $X Y$ plane at the middle draft of the DDS). 
225 To verify the numerical model, a mesh sensitivity study and a time step study were carried out. These

226 studies aimed to obtain the numerical results independent of mesh and time step variations. Details are

227 presented in Table 5 for a stationary DDS under $45^{\circ}$ flow incidence. Results for all cases are obtained

228 by averaging after more than fifteen vortex shedding cycles.

229

230

231

232

233

234

235

236

237

238

239

240

241

242

243

244

245

246

247

248

249

\subsection{Convergence Studies}

The convergence studies, based on the effects of mesh refinement and time step variations, provided results for time-averaged drag force coefficient $\left(\bar{C}_{D}\right)$ and Strouhal number $(S t)$. The Strouhal number ( $S t=f_{s} L / U_{c}$, where $f_{s}$ is the vortex shedding frequency, $L$ is the width of the column and $U_{c}$ is the free stream flow velocity) is obtained from the power spectra of the fluctuating lift force coefficient as suggested by Schewe [30].

The force coefficients $\left(C_{D}, C_{L}\right)$ are defined as:

$C_{D}=\frac{F_{D}}{\frac{1}{2} \rho U_{C}^{2} A}$

$$
C_{L}=\frac{F_{L}}{\frac{1}{2} \rho U_{C}^{2} A}
$$

where, $F_{D}$ is the drag force on the structure, $F_{L}$ is the lift force on the structure, $\rho$ is the fresh water density, $U_{C}$ is the free stream velocity and $A$ is the projected area.

Firstly, five tests at $R e=7.6 \times 10^{4}$ were carried out with different mesh especially in the near wake region of the structure. The DDS_M1 case is presented as relatively coarse mesh in the test. In this model, the computational domain consists of 0.15 million elements. Further cases ranged from 0.56 million to 6.86 million elements (see Table 5). All cases firstly used the same non-dimensional time step of 0.008 .

Finally, a time step sensitivity study was carried out for the convergence evaluation. The geometric model chosen for initial time step sensitivity study is the case DDS_M3, which was then repeated for two further cases with different time steps. A summary of these seven different sets of analyses is given in Table 5, and the results are presented in Table 6 and Table 7.

Table 5. Numerical set-up information. 


\begin{tabular}{lll}
\hline DDS_M1 & 0.15 & 0.008 \\
DDS_M2 & 0.56 & 0.008 \\
DDS_M3 & 3.43 & 0.008 \\
DDS_M4 & 5.08 & 0.008 \\
DDS_M5 & 6.86 & 0.008 \\
DDS_T1 & 3.43 & 0.016 \\
DDS_T2 & 3.43 & 0.004 \\
\hline
\end{tabular}

Table 6. The mesh refinement tests.

\begin{tabular}{llllll}
\hline Case & Elements (million) & $\overline{\boldsymbol{C}}_{\boldsymbol{D}}$ & $\begin{array}{l}\text { Relative } \\
\text { variation (\%) }\end{array}$ & $\boldsymbol{S t}$ & $\begin{array}{l}\text { Relative } \\
\text { variation (\%) }\end{array}$ \\
\hline DDS_M1 & 0.15 & 1.030 & & 0.122 & \\
DDS_M2 & 0.56 & 1.064 & 3.301 & 0.122 & 0 \\
DDS_M3 $^{*}$ & 3.43 & 1.068 & 0.376 & 0.131 & 7.377 \\
DDS_M4 & 5.08 & 1.075 & 0.655 & 0.131 & 0 \\
DDS_M5 & 6.86 & 1.066 & 0.837 & 0.131 & 0 \\
\hline
\end{tabular}

251 Table 7. The time step sensitivity study.

\begin{tabular}{llllll}
\hline Case & $\begin{array}{l}\text { Non-dimensional time step } \\
(\Delta \boldsymbol{U} \boldsymbol{U} / \boldsymbol{L})\end{array}$ & $\overline{\boldsymbol{C}}_{\boldsymbol{D}}$ & $\begin{array}{l}\text { Relative } \\
\text { variation (\%) }\end{array}$ & $\boldsymbol{S t}$ & $\begin{array}{l}\text { Relative } \\
\text { variation }(\%)\end{array}$ \\
\hline DDS_T1 & 0.016 & 1.020 & & 0.131 & \\
DDS_M3 $^{*}$ & 0.008 & 1.068 & 4.706 & 0.122 & 11.486 \\
DDS_T2 & 0.004 & 1.068 & 0 & 0.122 & 0 \\
\hline
\end{tabular}

252 As shown in Table 6 and Table 7, the DDS_M3 case is considered to be fine enough for both mesh

253 refinement effects and a suitable time step. Case DDS_M3 has been chosen for the further validation

254 of the numerical model against experimental data. 

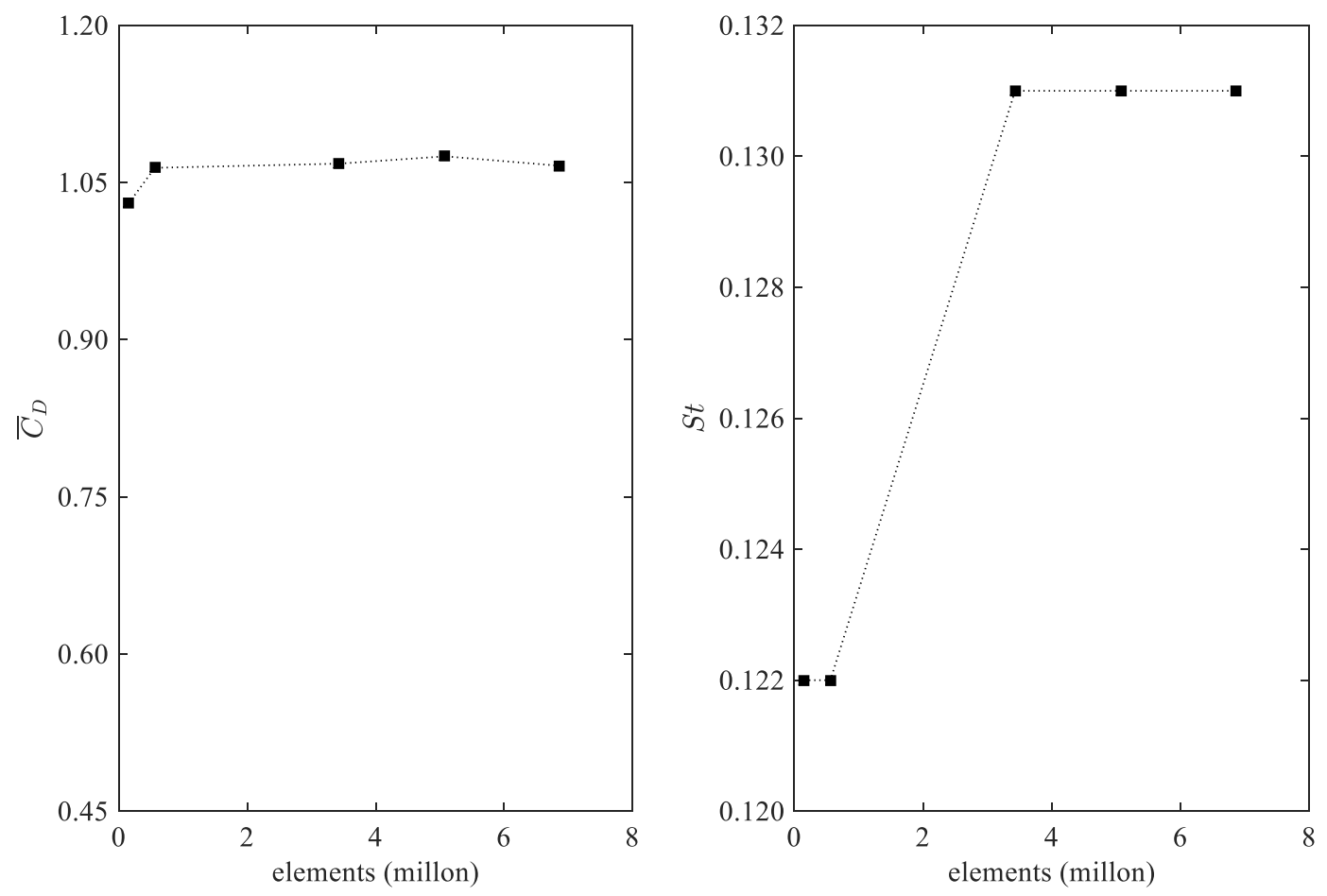

256 Fig. 6. Convergence line for both $\bar{C}_{D}$ and St.

\section{3.3. Model validation}

258 Experimental data available from MARIN [14] is the main reference for validating the present

259 numerical simulation. The resulting $\bar{C}_{D}$ from the present numerical calculation and the experimental

260 measurements are presented in Table 8.

261 Table 8. Comparison of $\bar{C}_{D}$ from the present numerical calculation and the MARIN experimental measurements.

\begin{tabular}{ll|l}
\hline Case & $\overline{\boldsymbol{C}}_{\boldsymbol{D}}$ & Relative variation (\%) \\
\cline { 1 - 2 } Present numerical model & 1.127 & \multirow{2}{*}{8.78} \\
\cline { 1 - 2 } MARIN test [14] & 1.036 & \\
\hline
\end{tabular}

263 Compared with the experimental data, the results from the present numerical model show good 264 agreement with a relative variation $8.78 \%$. Therefore, the present numerical model can be used with confidence in future VIM motion simulations.

266 Table 9. Comparison of results from the present numerical calculations and experimental measurements 267 for $45^{\circ}$ incidence. 


\begin{tabular}{|c|c|c|c|c|c|c|c|c|c|}
\hline$U r$ & $\begin{array}{l}\bar{C}_{D} \\
\text { (num.) }\end{array}$ & $\begin{array}{l}\bar{C}_{D} \\
(\text { exp. })\end{array}$ & $\begin{array}{l}\text { Relative } \\
\text { variation } \\
(\%)\end{array}$ & $\begin{array}{l}C_{\text {Lrms }} \\
\text { (num.) }\end{array}$ & $\begin{array}{l}C_{\text {Lrms }} \\
\text { (exp.) }\end{array}$ & $\begin{array}{l}\text { Relative } \\
\text { variation } \\
(\%)\end{array}$ & $\begin{array}{l}A_{y} / L \\
\text { (num.) }\end{array}$ & $\begin{array}{c}A_{y} / L \\
(\text { exp.) }\end{array}$ & $\begin{array}{l}\text { Relative } \\
\text { variation } \\
(\%)\end{array}$ \\
\hline 3.9 & 2.210 & 2.399 & 7.9 & 0.931 & 0.751 & 23.9 & 0.236 & 0.166 & 41.9 \\
\hline 6.6 & 2.619 & 2.614 & 0.2 & 0.540 & 0.497 & 8.5 & 0.760 & 0.742 & 2.5 \\
\hline 8.9 & 2.292 & 2.429 & 5.7 & 0.190 & 0.230 & 17.3 & 0.378 & 0.398 & 5.0 \\
\hline 12.1 & 2.099 & 2.154 & 2.6 & 0.194 & 0.177 & 9.8 & 0.345 & 0.318 & 8.5 \\
\hline
\end{tabular}

268

269

270

271

272

273

274

275

276

277

278

279

280

281

282

283

284

285

286

287

288

289

290

291

292

In addition to the experimental data from MARIN, further comparison of $\bar{C}_{D}, C_{L r m s}$ and $A_{y} / L$ with the present experimental and numerical investigations are provided in Table 9 showing good agreements, hence providing another means of validating the numerical model in this study. Most of the relative variations are less than $10 \%$, especially for the predictions of $\bar{C}_{D}$. However, it is noted that at $U r=3.9$, the numerical results have a relatively large discrepancy when compared with the experimental data. The detailed discussions will be presented in 4.2. Force analysis.

\section{Results and discussion}

The motion response of a typical moored DDS under four current velocities for each of the two headings were investigated using the present numerical model and their results are further compared with the measurements conducted in the towing tank. The motion measurements for more than ten cycles of the VIM oscillation period are collected in the present study.

\subsection{Motion characteristics}

\subsubsection{Motions in transverse and in-line directions}

Fig. 7, which compares the results from the numerical simulation with those from the model tests, presents the non-dimensional characteristic amplitude $\left(A_{x} / L, A_{y} / L\right.$, where $U r$ is defined based on $T_{\text {Otransverse }}$ ) for motions in both the in-line and the transverse directions under flow incidences of $0^{\circ}$ and $45^{\circ}$. The non-dimensional amplitude is defined as $\sqrt{2} \times \sigma\left(\frac{y(t)}{L}\right)$ (where $\sigma$ is the standard deviation of the time series $y(t) / L$, and $y(t)$ represents the time series of in-line, transverse and yaw motions. For yaw motions the non-dimensional amplitude is defined as $\sqrt{2} \times \sigma(y(t)))$. As can be seen in Fig. 7, the $45^{\circ}$ incidence cases showed larger VIM in magnitude in both the in-line and the transverse directions. Moreover, the largest $A_{x} / L$ for both incidences occurred at the same range around $U r=6.5$. The "lock-in" region for $0^{\circ}$ incidence happens in the range of $6.0 \leq U r \leq 9.5$, while for $45^{\circ}$ incidence, the "lock-in" region occurs between $5.0 \leq U r \leq 9.0$. It is also to be noted that the motion in the in-line direction for both incidences keeps increasing with increasing $U r$. In this context, the present numerical model predicts the motions in both the transverse and the in-line directions well. At low $U r$ 
values, the numerical model predicts a larger response than the experimental results in the transverse direction. When the towing speed for $U r$ is extremely low $\left(0.062 \mathrm{~m} / \mathrm{s}\right.$ for $0^{\circ}$ incidence at $\left.U r=3.9\right)$ in the towing tank test, it is likely that the "friction" of the whole physical facilities tends to affect the experimental measurements. However, at high $U r$, such effect become insignificant, especially in the "lock-in" region where the numerical predictions agree well with the experimental data. Fig. 8 and Fig. 9 present the results of the motion in the transverse direction for $0^{\circ}$ and $45^{\circ}$ flow incidences in the frequency domain. It is clearly seen that the motion responses very much concentrate around the natural transverse frequency $\left(f_{\text {Otransverse }}\right)$ in the "lock-in" region. Moreover, as can be seen in Fig. 10 and Fig. 11, both incidences show that in the "lock-in" region, the structure's response frequency $\left(f_{y}\right)$ is approximately the same as the vortex shedding frequency $\left(f_{s}\right)$ (also seen in Fig. 12 and Fig. 13). It is further observed in both experiments and numerical simulations that, for $0^{\circ}$ incidence, in the "post lock-in" region $f_{s}$ increases and becomes larger than $f_{y}$. This phenomenon was also observed by Waals et al. [14] and termed as galloping. Galloping is different from VIM. It describes a low frequency response and is not self-limiting. When Galloping happens, $f_{s}$ is much larger than the structural response frequency [14]. The transverse motion tends to increase with increasing $U r$. This can be found in Fig. 8 and Fig. 10. In the "post lock-in" region, the peak $f_{y}$ is still around $f_{\text {Otransverse }}$ Additionally, a high $f_{y}$ appeared with increasing $U r$ (see Fig. 8). However, in the vortex shedding frequency domain, at $U r=15.7$, there are two nearly equal weight peak transverse forces occurring at two vortex shedding frequencies (as shown in Fig. 14b). In addition, it is more clear that $f_{s}$ is three times as the peak $f_{y}$ at $U r=20.2$ in the experimental measurements (see Fig. 8 and Fig. 10). Thus, the motion response in the "post lock-in" region is an oscillation phenomenon which combines VIM and the galloping phenomena. In this situation, $A_{x} / L$ keeps increasing in the "post lock-in" region. 

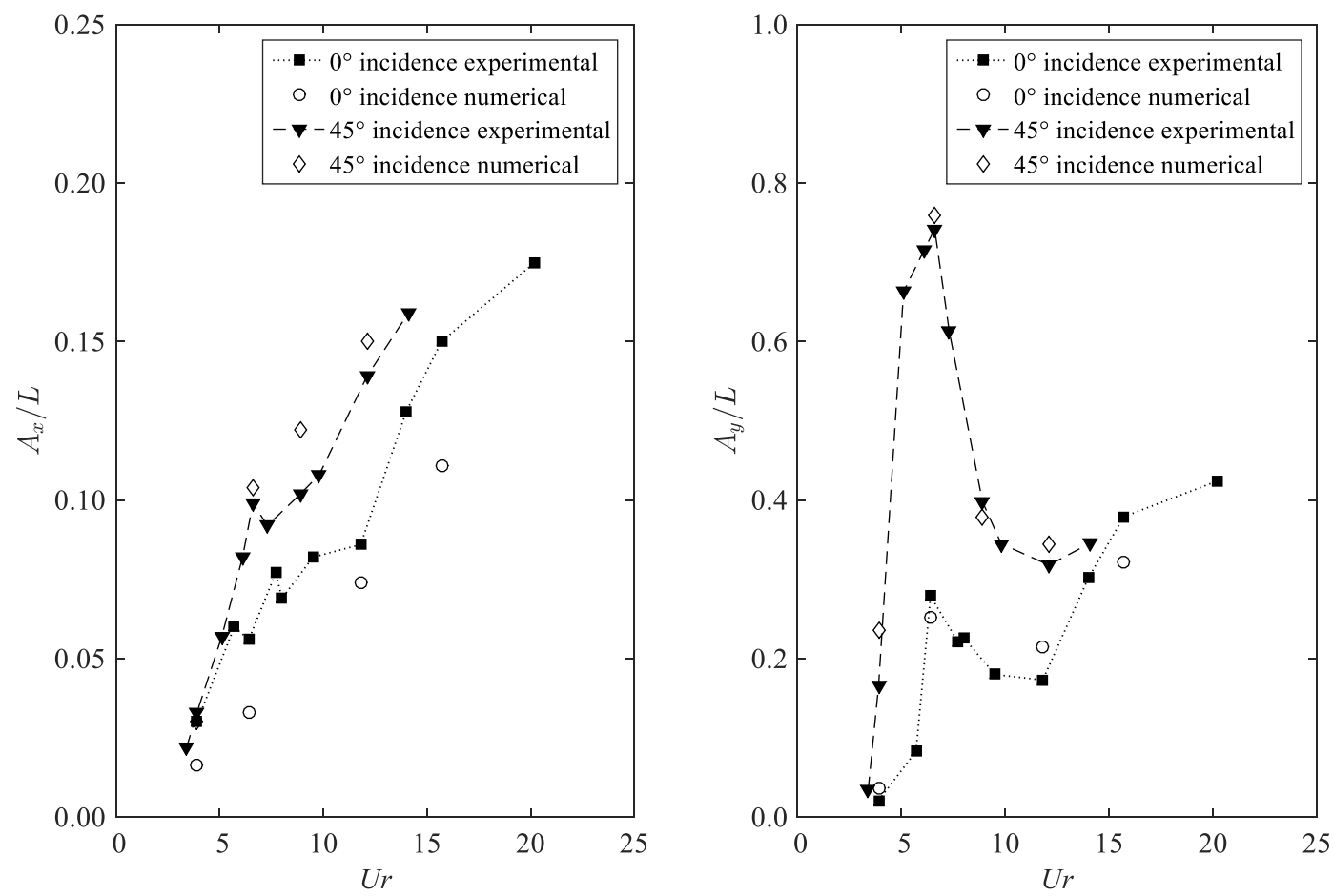

315

316 Fig. 7. Non-dimensional in-line and transverse characteristics amplitudes $\left(A_{x} / L, A_{y} / L\right)$, the $U r$ is

317 defined based on $\left.T_{\text {Dtransverse }}\right)$.

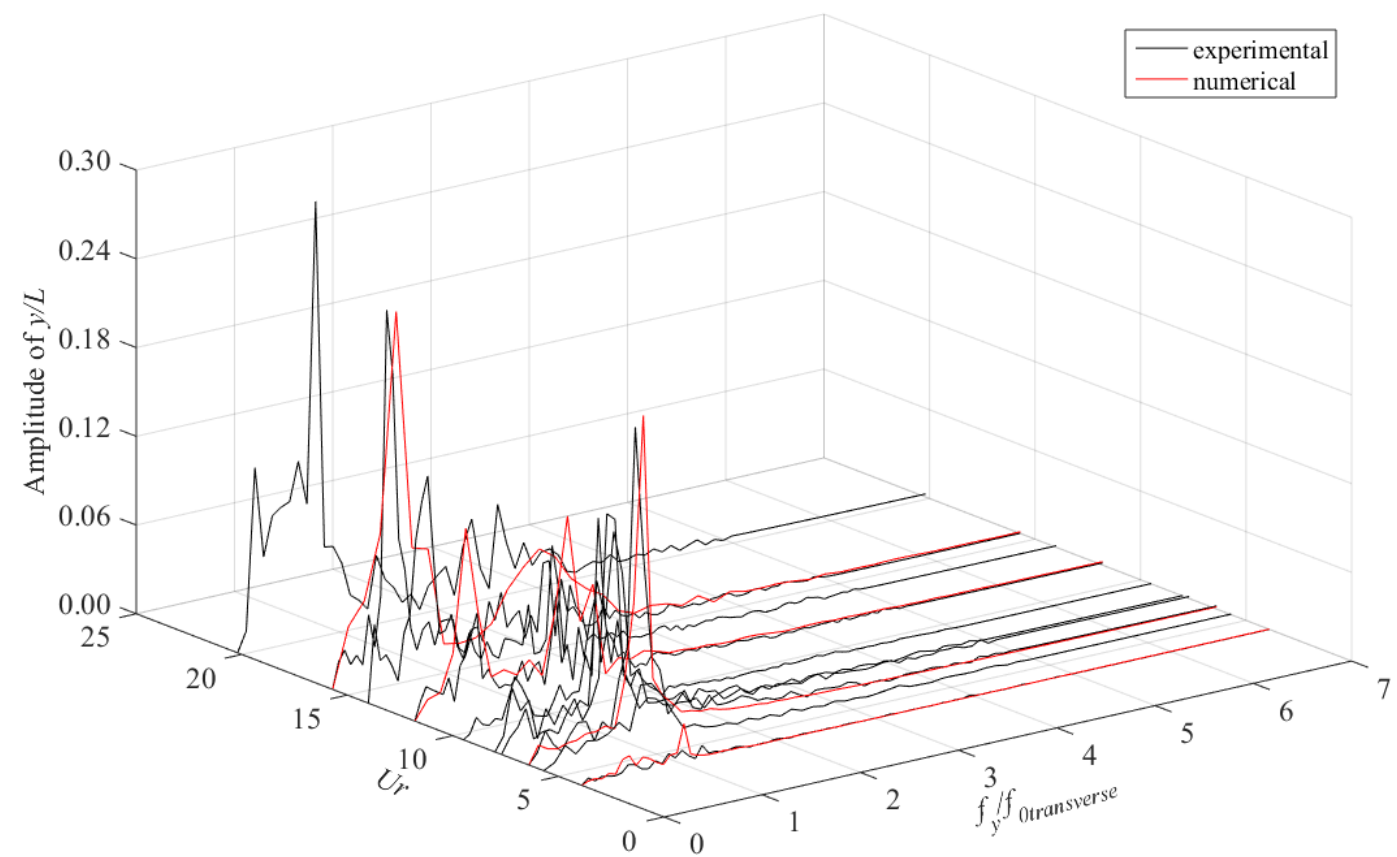



$U r$ is defined based on $\left.T_{\text {transverse }}\right)$.

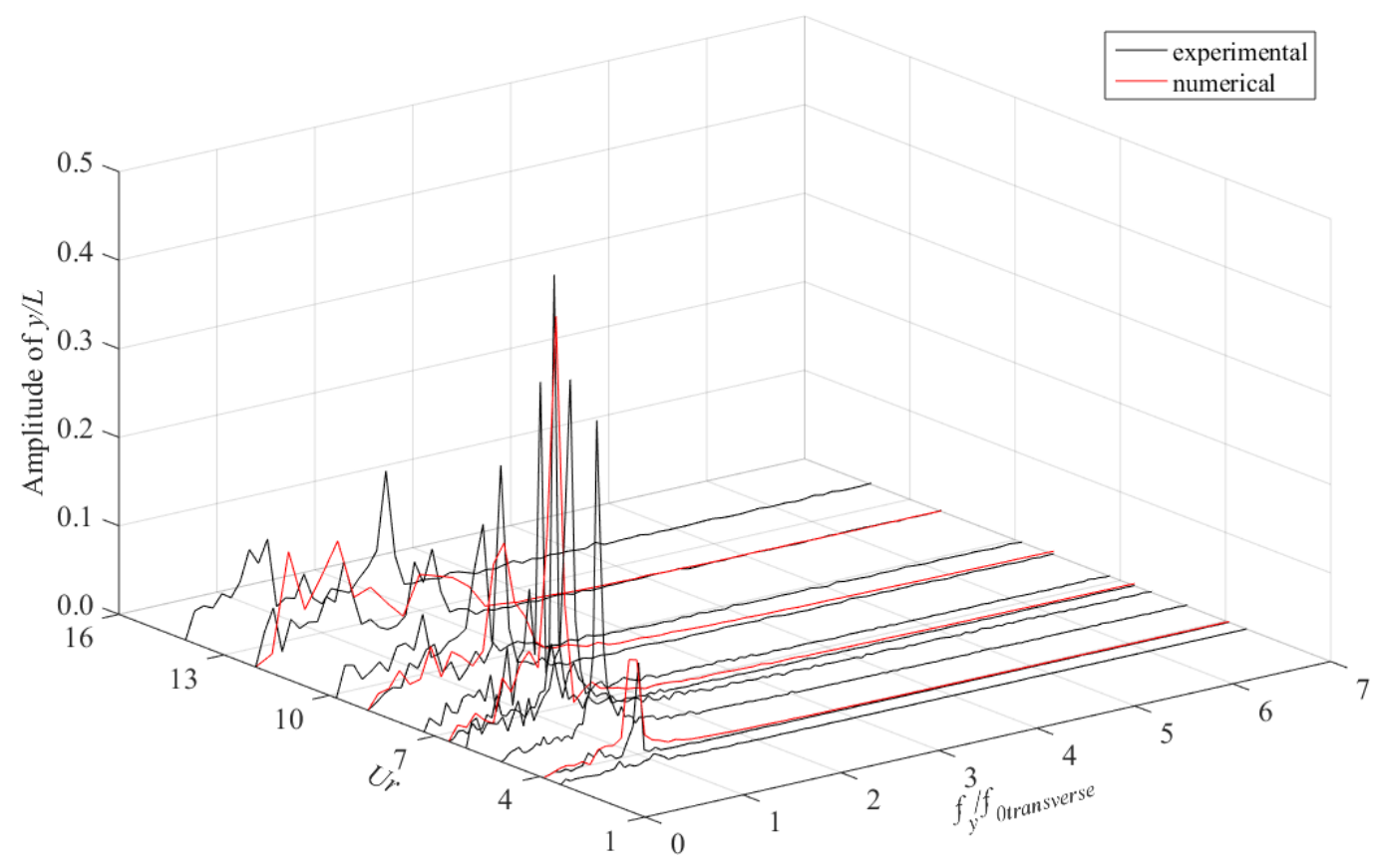

322 Fig. 9. FFT of the motions in the transverse direction as a function of $U r$ and $f_{y}$ for $45^{\circ}$ incidence (the $U r$ is defined based on $T_{\text {transverse }}$ ). 


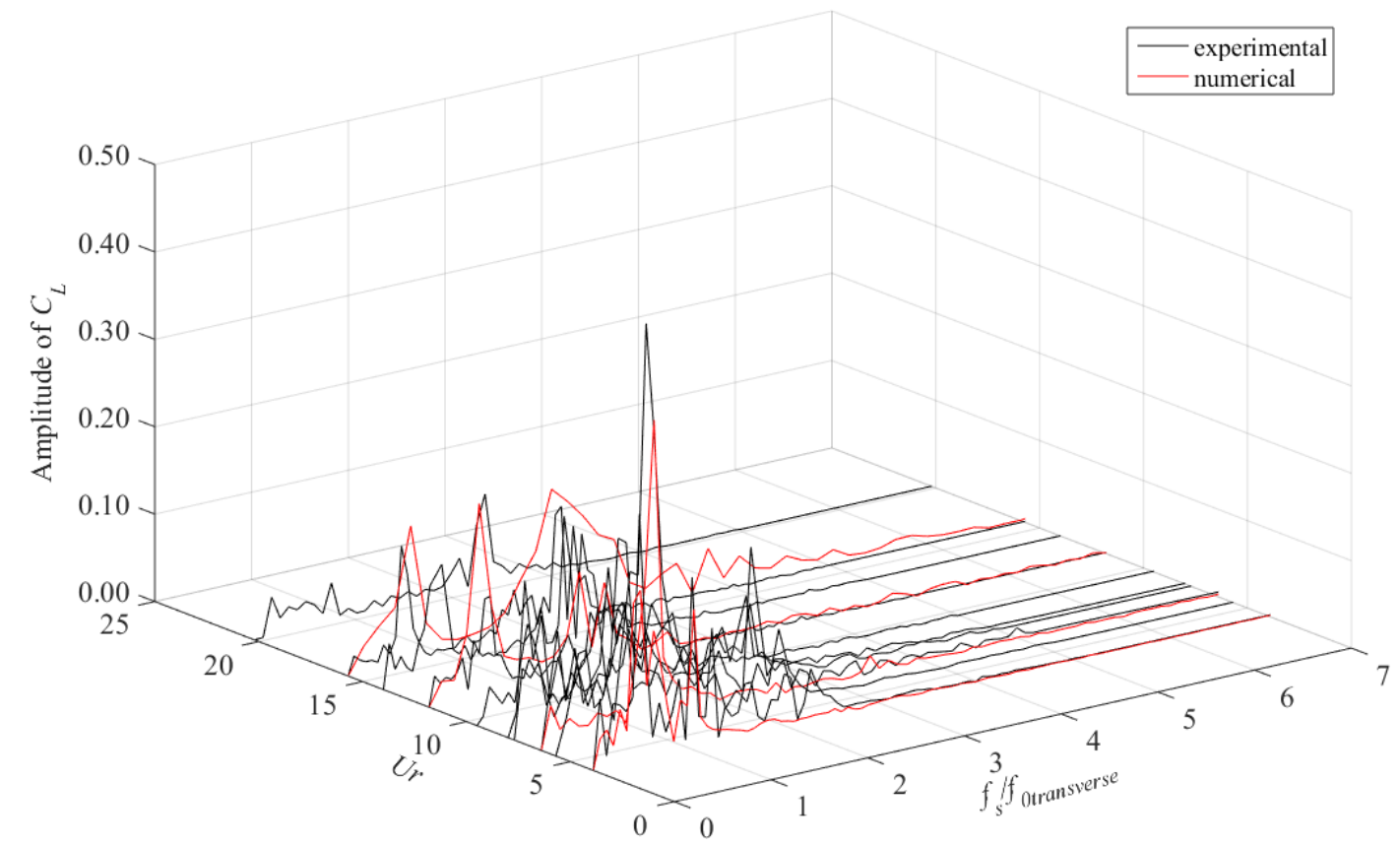

325 Fig. 10. FFT of lift force coefficient as a function of $U r$ and $f_{s}$ for $0^{\circ}$ incidence (the $U r$ is defined 326 based on $T_{\text {Otransverse }}$ ).

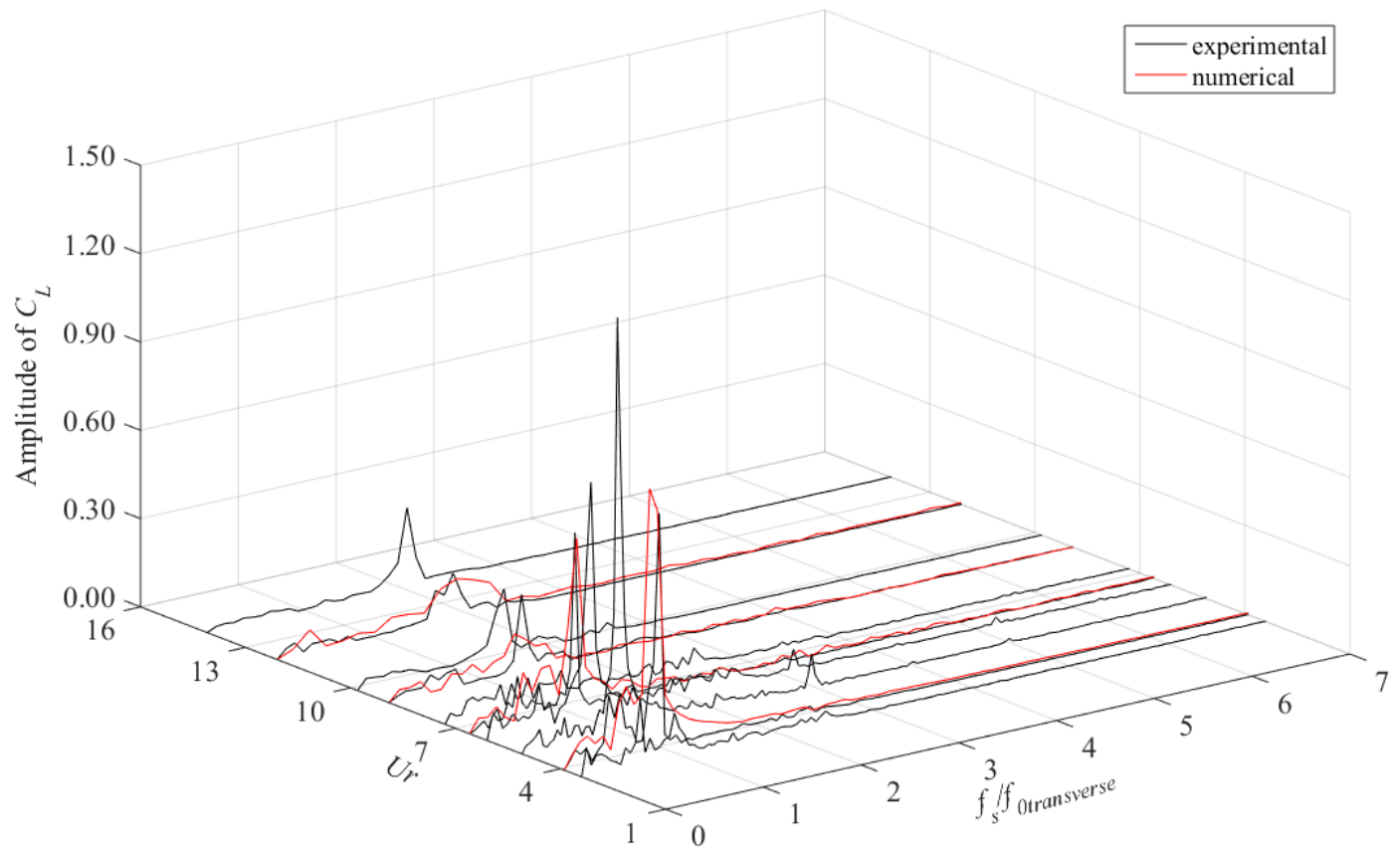


Fig. 11. FFT of lift force coefficient as a function of $U r$ and $f_{s}$ for $45^{\circ}$ incidence (the $U r$ is defined based on $\left.T_{\text {Otransverse }}\right)$.
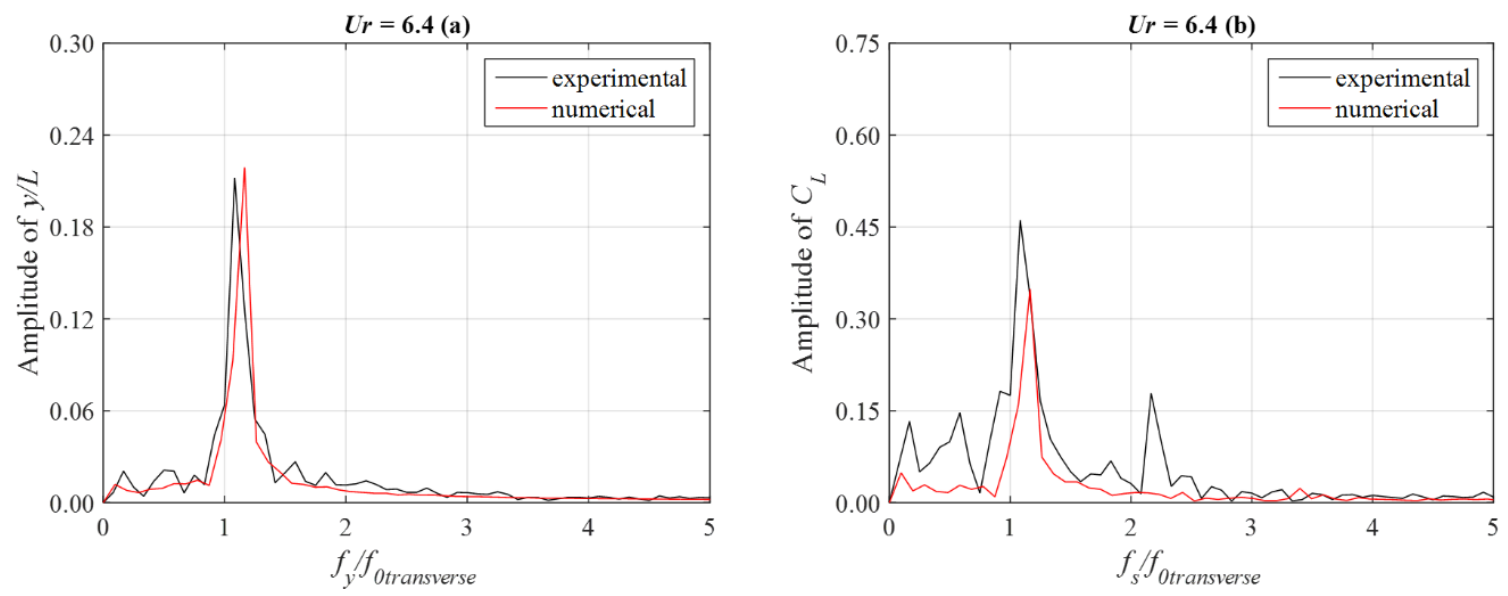

331 Fig. 12. FFT of the transverse motions and the lift force coefficients at $U r=6.4$ for $0^{\circ}$ incidence, (a) transverse motion $(y / L)$; (b) lift force coefficient $\left(C_{L}\right)$.
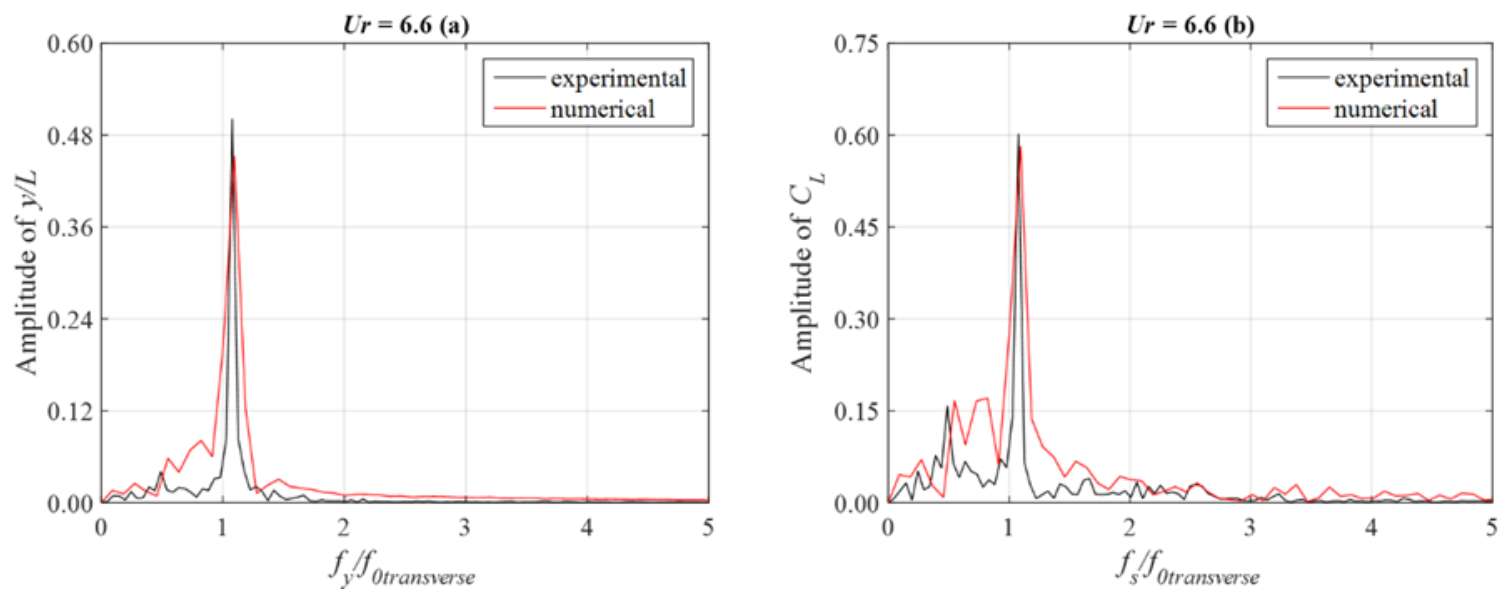

334 Fig. 13. FFT of the transverse motions and the lift force coefficients at $U r=6.6$ for $45^{\circ}$ incidence, (a) transverse motion $(y / L)$; (b) lift force coefficient $\left(C_{L}\right)$. 

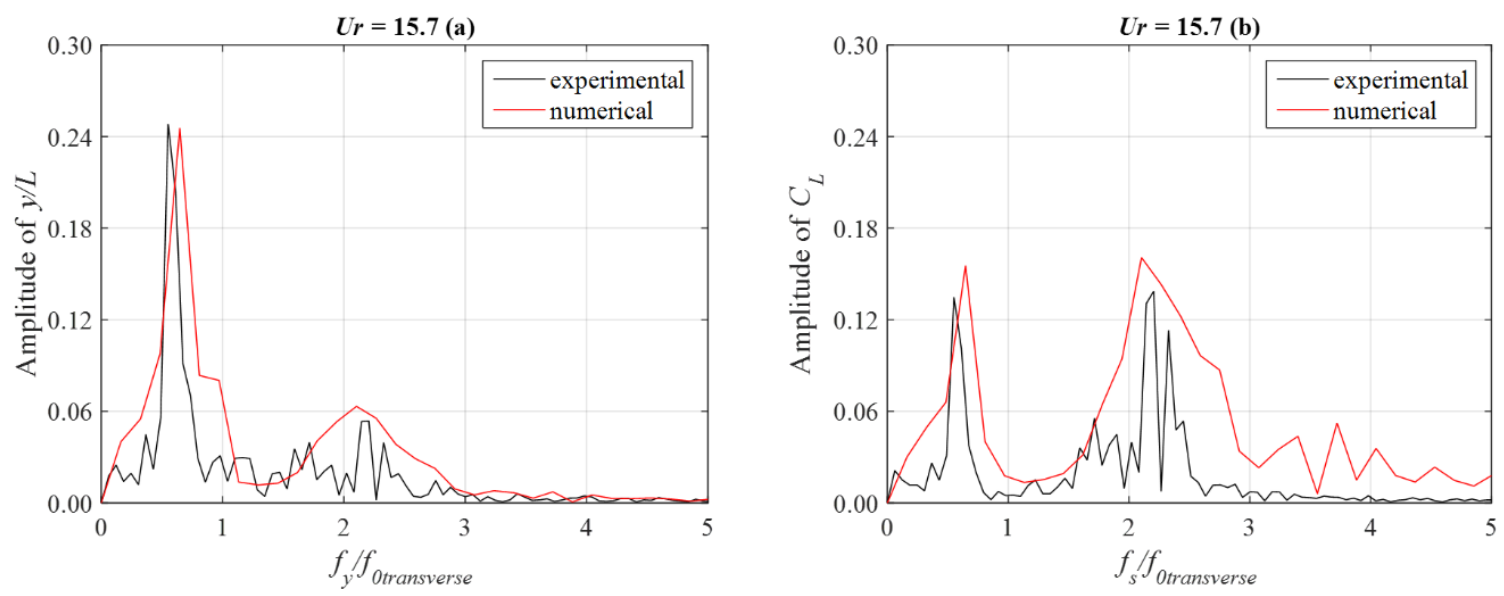

337 Fig. 14. FFT of the transverse motions and the lift force coefficients at $U r=15.7$ for $0^{\circ}$ incidence, (a)

338 transverse motion $(y / L)$; (b) lift force coefficient $\left(C_{L}\right)$.

339 It is noted that the transverse motions are somewhat sinusoidal with near constant amplitude as would 340 be expected at "lock-in". However, in the "pre lock-in" and "post lock-in" region, the time history of 341 the non-dimensional transverse amplitude shows considerable variability. Thus, a new variable called 342 the significant value of transverse peaks $\left(A_{1 / 3} / L\right)$ is introduced in the present study in order to examine 343 the irregular characteristics of the transverse motions.

$344 \quad A_{1 / 3} / L=\frac{1}{\frac{1}{3} N} \sum_{m=1}^{\frac{1}{3} N} A_{m} / L$

345 where $N$ is the number of the oscillations and $L$ is the columns' width, the largest $A_{m}$ has $m=1$ and the 346 lowest $A_{m}$ is for $m=N, A_{m}$ is the individual oscillation height which is defined as:

$347 \quad A_{m}=A_{\text {peak }}-A_{\text {bottom }}$

348 where $A_{\text {peak }}$ and $A_{b o t t o m}$ are the points when $\frac{d y}{d t}=0$ within one oscillation period. 


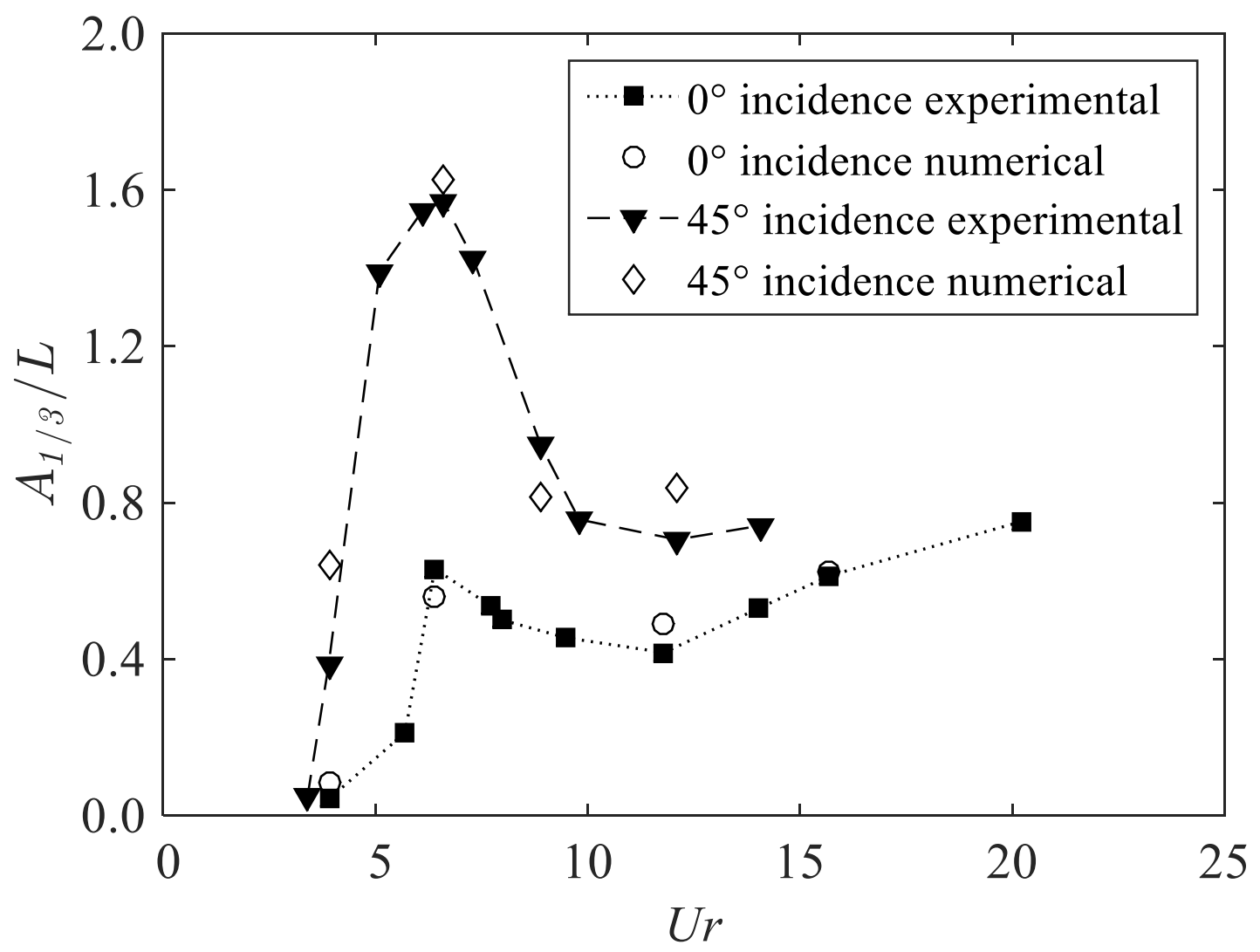

Fig. 15. Significant values of the transverse peaks $\left(A_{1 / 3} / L\right)$. The $U r$ is defined based on $T_{0 \text { transverse }}$.

Fig. 15 presents $A_{1 / 3} / L$ as a function of $U r$. It shows that, in the "pre lock-in" and "post lock-in" region, the present numerical model predicts the characteristics of the transverse motions well when compared with the experimental results.

\subsubsection{Yaw motions}

Fig. 16 presents the non-dimensional yaw amplitude. Fig. 17 and Fig. 18 present the yaw motions in the frequency domain as a function of $U r$. In the current study, it is observed that the in-line, transverse and yaw natural frequencies are relatively close to each other. The non-dimensional amplitude for the $0^{\circ}$ incidence yaw motion shows the same trend as $A_{y} / L$. However, for $45^{\circ}$ incidence, the yaw motion response is different from the transverse motion response. In the "lock-in" region, the non-dimensional yaw amplitudes at $0^{\circ}$ incidence are larger than those at $45^{\circ}$ incidence. For both flow incidences, the numerical method predicts the motion response trend well comparing with the experimental results. It is noted that in the numerical simulations, the mooring lines are idealised springs' arrangement which are exactly symmetrical. However, in the experiments, slight differences 
can be observed in the forces on each side of the mooring lines possibly owning to the mooring lines being not exactly the same and the effects of spring bending due to the gravity on springs in the experimental set-up. The slight differences on the mooring lines causes the structure to have a small attack angle with the current flow during VIM. Especially for $0^{\circ}$ incidence, the small attack angle makes the columns at the balance position not exactly perpendicular to the current leading to a slight variation in the hydrodynamic moment measurements. This may contribute to the discrepancies between the numerical predictions and the experimental data for the yaw motion.

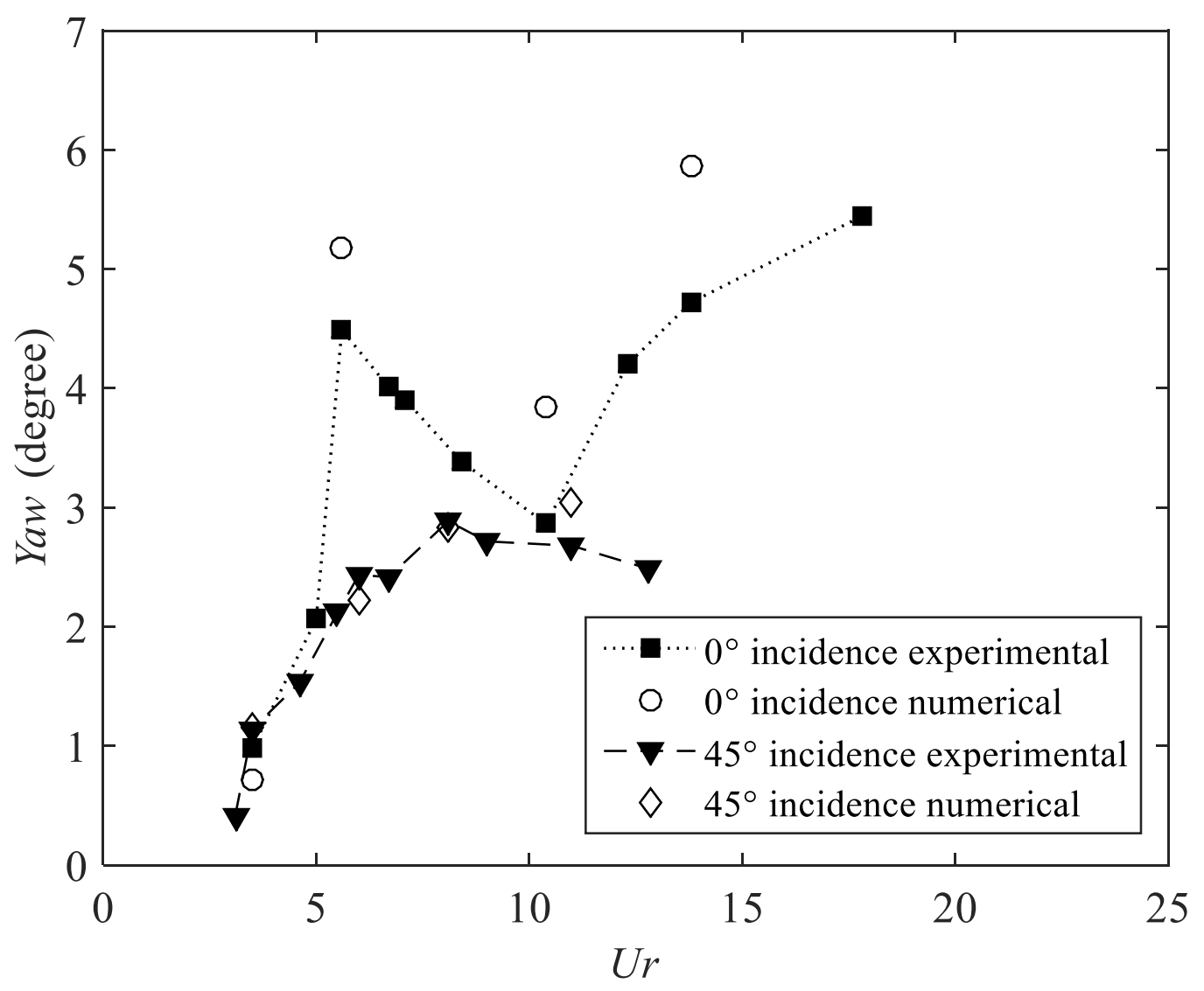

371

372 Fig. 16. Non-dimensional yaw characteristics amplitude (the $U r$ is defined based on $T_{\text {0yaw }}$ ). 


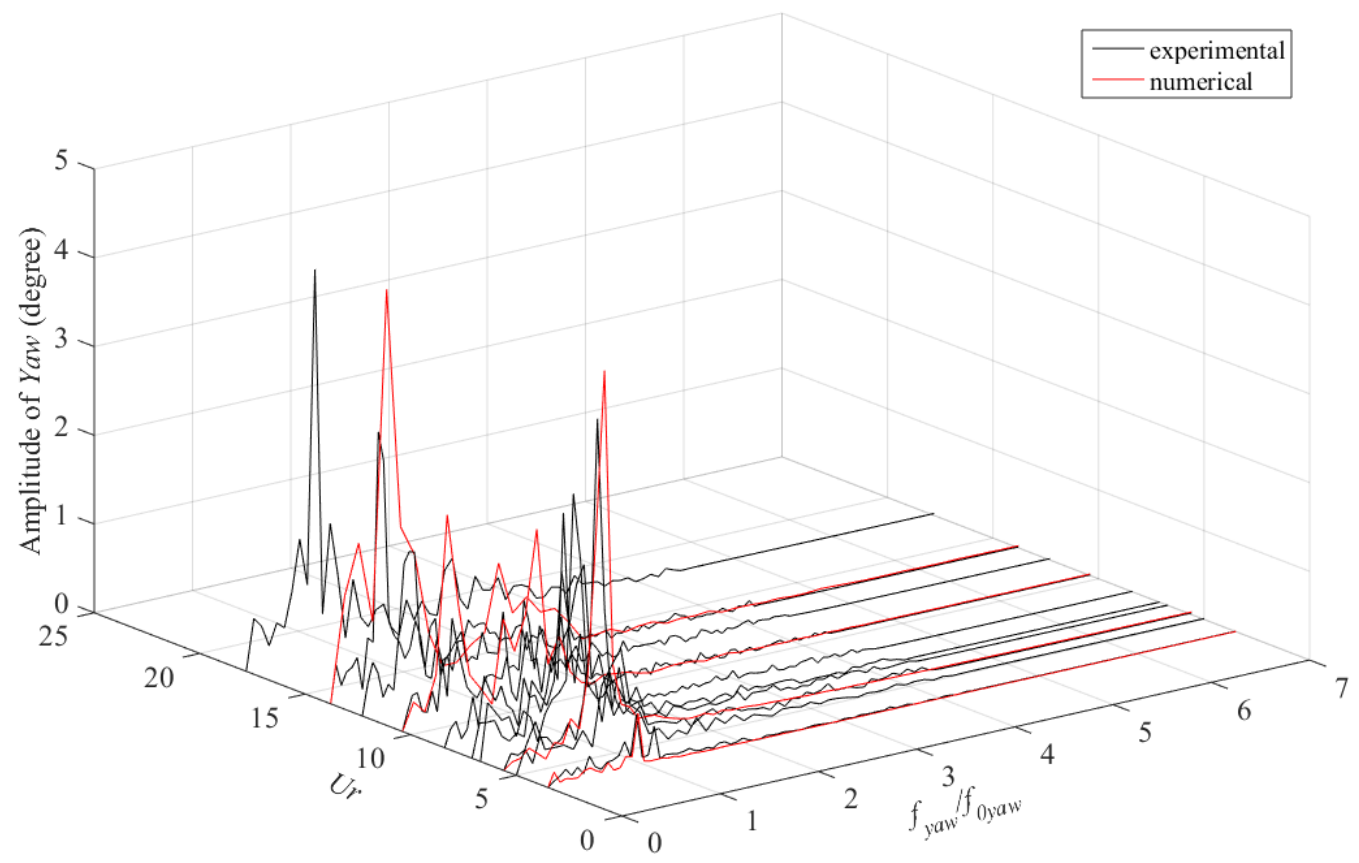

373

374 Fig. 17. FFT of the yaw motion as a function of $U r$ and $f_{\text {yaw }}$ for $0^{\circ}$ incidence (the $U r$ is defined based 375 on $\left.T_{\text {Oyaw }}\right)$.

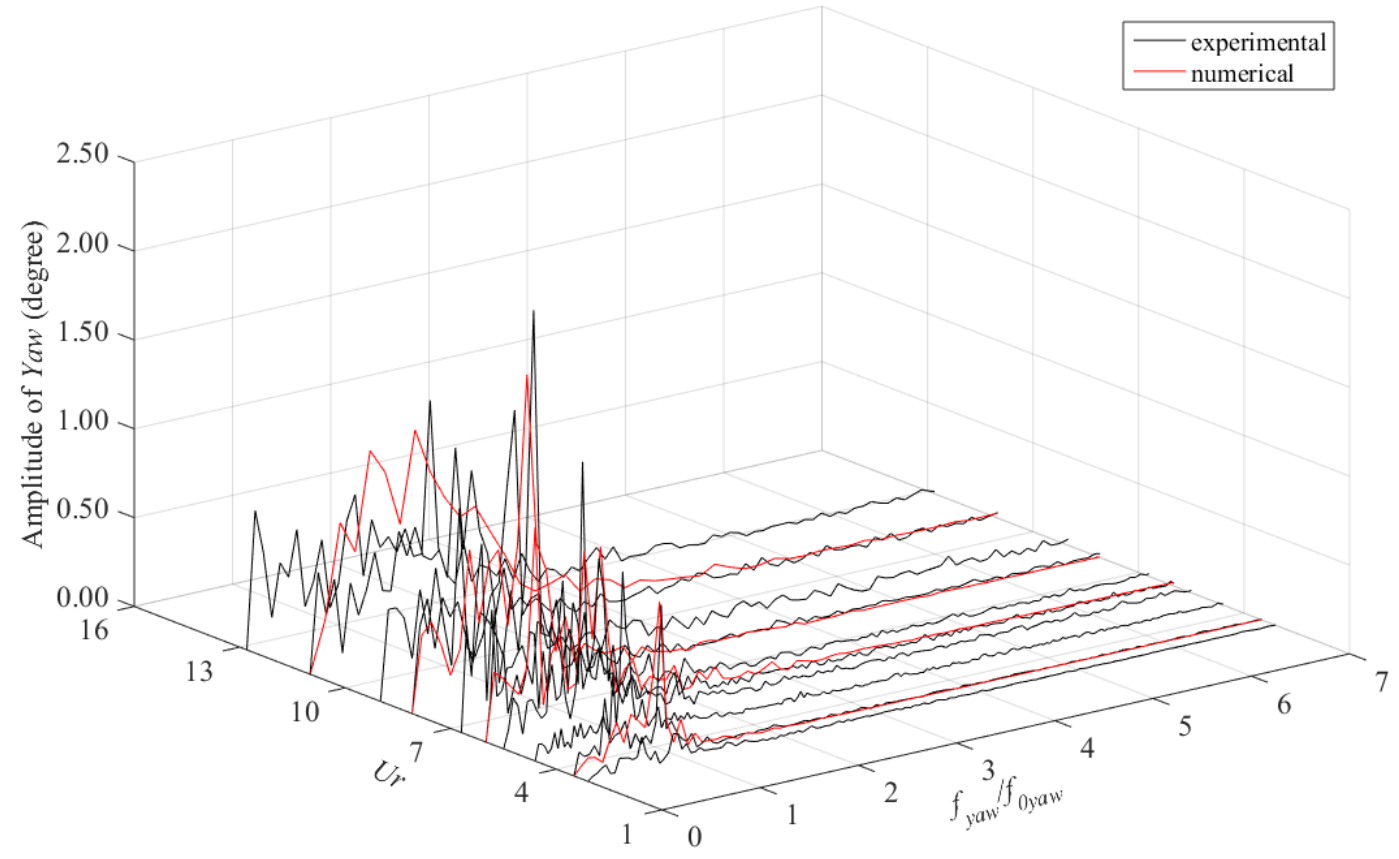


377 Fig. 18. FFT of the yaw motion as a function of $U r$ and $f_{\text {yaw }}$ for $45^{\circ}$ incidence (the $U r$ is defined based 378 on $\left.T_{\text {Oyaw }}\right)$.

380 The fluid forces on the structure are calculated by the equation given by Sarpkaya [31] as:

$381 m \ddot{X}(t)+C \dot{X}(t)+K_{x}(t)=F_{x}(t)$

$382 m \ddot{Y}(t)+C \dot{Y}(t)+K_{y}(t)=F_{y}(t)$

383 where $m$ is the platform mass; $C$ is the structural damping coefficient; $K_{x}$ and $K_{y}$ are the linear spring 384 constant in the in-line and transverse directions; $F_{x}(t)$ and $F_{y}(t)$ represent the in-line and transverse 385 hydrodynamic forces acting on the structures.

386 As the structural damping coefficient is very small and can be disregarded. The hydrodynamic forces 387 which include added mass and hydrodynamic damping forces due to fluid are placed on the right side 388 of the equations. Thus, the Equation (6) and (7) can be written as:

$389 m \ddot{X}(t)+K_{x}(t)=F_{x}(t)$

$390 \quad m \ddot{Y}(t)+K_{y}(t)=F_{y}(t)$

391 In the present study, the total hydrodynamic forces in the experiments are measured indirectly by

392 using the equations given by Sarpkaya [31]. However, the total hydrodynamic forces of the numerical 393 predictions are obtained directly from the CFD simulations. 


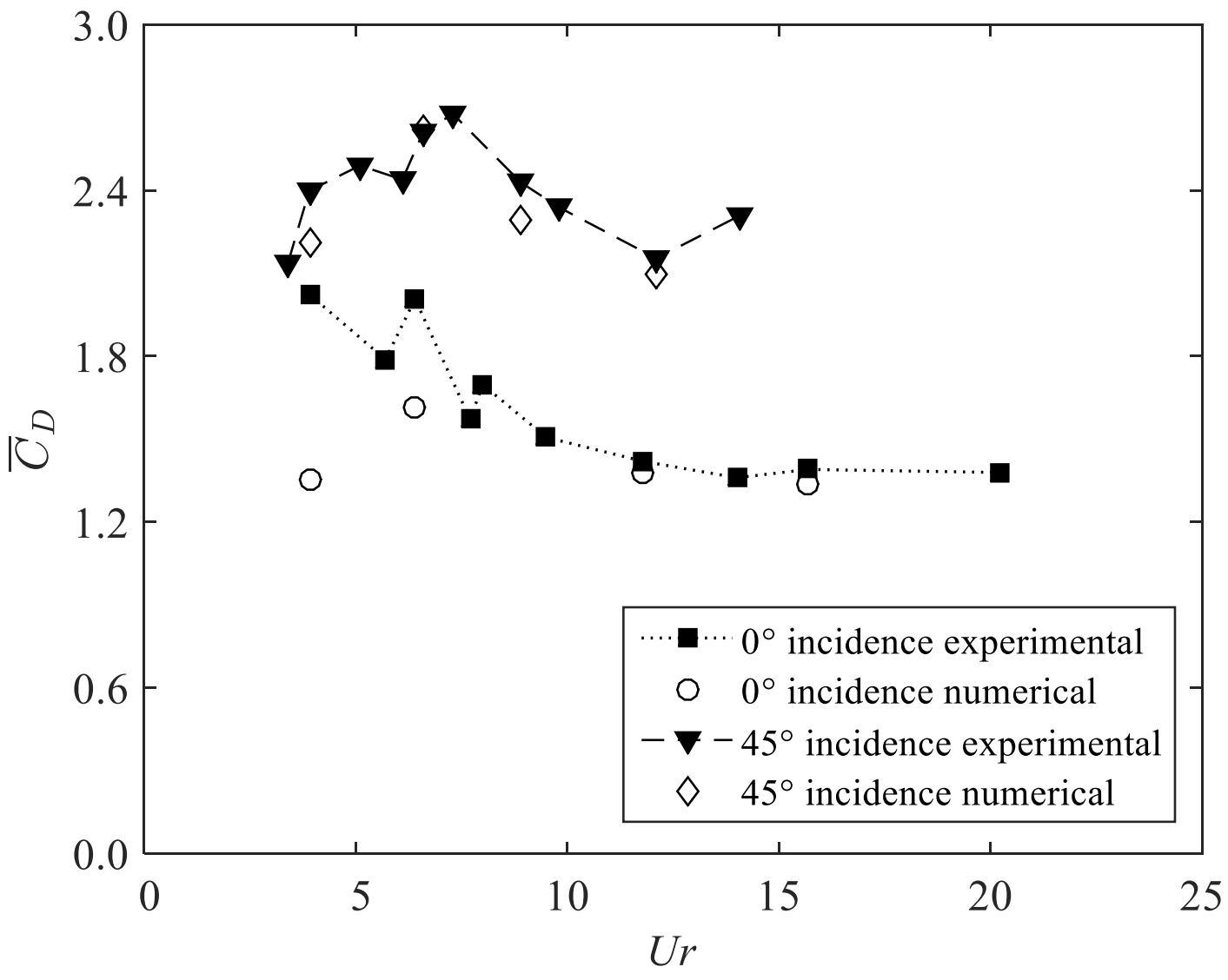

395 Fig. 19. Mean drag coefficient $\left(\bar{C}_{D}\right)$, where $A$ is the projected area at $0^{\circ}$ incidence.

396 The drag coefficients for both $0^{\circ}$ and $45^{\circ}$ flow incidences are shown in Fig. 19. For $0^{\circ}$ incidence, the numerical results show a large discrepancy to experimental measurements at low $U r$. This is possibly due to the extremely low towing speed $(0.062 \mathrm{~m} / \mathrm{s}$ for reduced velocity at $U r=3.9)$ in the experiment where the mechanical friction in the system set-up affects the force measurements especially at the very low towing speed. In addition, the mooring lines can have the most striking effects on the results. To investigate this further, the mooring line forces have been examined in both the experiment measurement and the numerical simulation. As shown in Table 10, the numerical predictions show that the forces on the mooring lines are symmetrical along the in-line direction. However, in the experiments, slight differences can be observed in the forces on each sides of the mooring lines possibly owning to the mooring lines being not exactly same and the effects of spring bending by the gravity in the experimental set-up. The differences of the mooring force between the upstream mooring lines (Mooring line 1 and Mooring line 2) are smaller than the downstream mooring lines

408 (Mooring line 3 and Mooring line 4). The asymmetrical forces on the mooring lines make the motions 409 of the structure asymmetrical. Especially for $0^{\circ}$ incidences cases, the asymmetrical forces on the mooring line make the structure to have a small attack angle with the current flow. This rotational 
411 offset is the main contributor to the differences between numerical predictions and experimental data.

412 When the $U r$ increases, the offset of the platform relative to the in-line direction also increases leading

413 to the forces on the downstream mooring line decreasing. Consequently, the effect of the force

414 difference in the downstream mooring lines is weakened with increasing $U r$. Therefore, the numerical

415 results agree well with experimental measurements for high $U r$ for $0^{\circ}$ incidence. As shown in Fig. 19,

$416 \bar{C}_{D}$ increases when "lock-in" occurs. This is because the fluctuations of the force on the structure is

417 excited by resonance. As can be seen in Fig. $19, \bar{C}_{D}$ at $45^{\circ}$ incidence is higher than that at $0^{\circ}$

418 incidence. Similar observation was also reported by Sumer and Fredsøe [32] for flow past a sharp-

419 corner square cylinder.

420 Table 10. Comparison of the mooring line mean forces for $0^{\circ}$ incidence at $U r=3.9,6.4$ (The mooring

421 lines arrangement is shown in Fig. 3).

\begin{tabular}{lllll}
\hline Mean mooring force & $\begin{array}{l}\text { Mooring line } \\
\mathbf{1}(\mathbf{N})\end{array}$ & $\begin{array}{l}\text { Mooring line } \\
\mathbf{2}(\mathbf{N})\end{array}$ & $\begin{array}{l}\text { Mooring line } \\
\mathbf{3}(\mathbf{N})\end{array}$ & $\begin{array}{l}\text { Mooring line 4 } \\
(\mathbf{N})\end{array}$ \\
\hline $\boldsymbol{U} \boldsymbol{r}=\mathbf{3 . 9}$ numerical & 7.365 & 7.375 & 6.425 & 6.415 \\
\hline $\boldsymbol{U} \boldsymbol{r}=\mathbf{3 . 9}$ experimental & 7.489 & 7.745 & 6.445 & 5.896 \\
\hline $\boldsymbol{U} \boldsymbol{r}=\mathbf{6 . 4}$ numerical & 8.481 & 8.405 & 5.523 & 5.601 \\
\hline $\boldsymbol{U} \boldsymbol{r}=\mathbf{6 . 4}$ experimental & 8.617 & 8.934 & 5.417 & 4.859 \\
\hline
\end{tabular}

\subsection{Vortex shedding characteristics}

423 To have a general visual appreciation of the vortex shedding pattern, the vorticity magnitude contours

424 obtained from the numerical simulations for "pre lock-in", "lock-in" and "post lock-in" regions are 425 plotted in Fig. 20. 
$0^{\circ}$ incidence

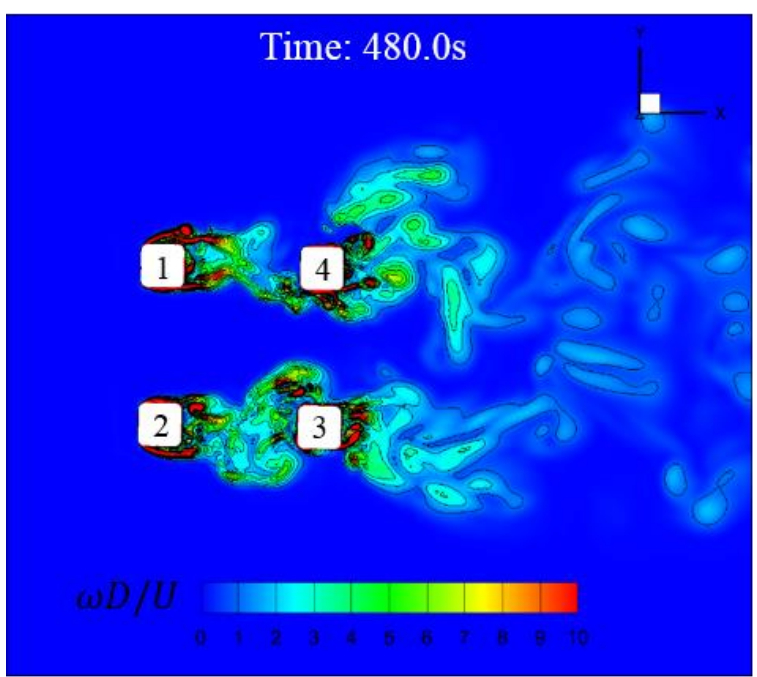

a) "pre lock-in" $(U r=3.9)$

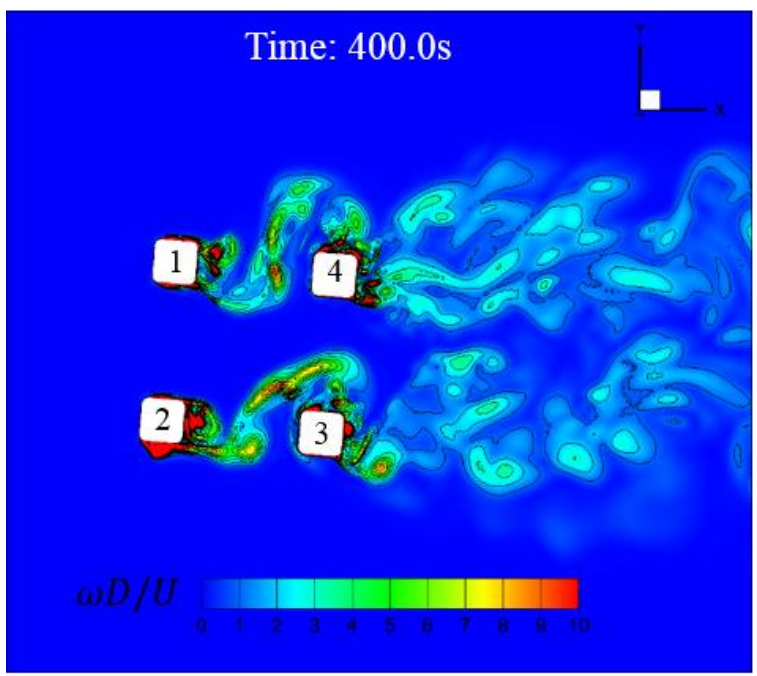

b) "lock-in" ( $U r=6.4)$

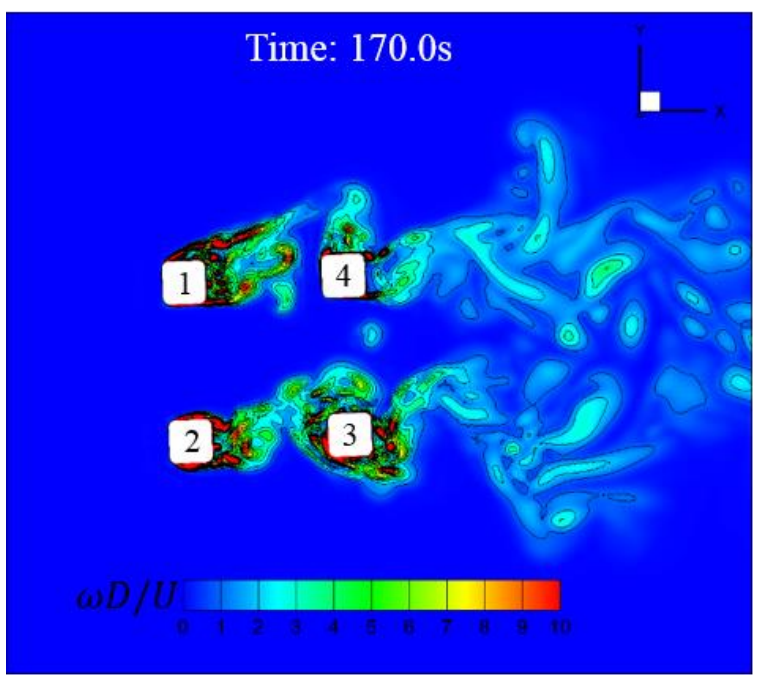

c) "post lock-in" $(U r=11.8)$

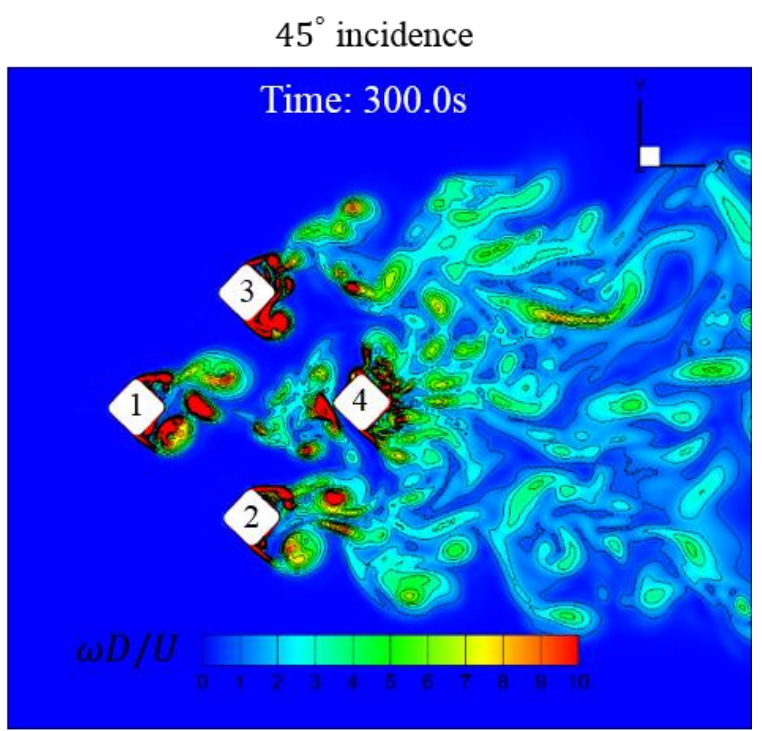

d) "pre lock-in" $(U r=3.9)$

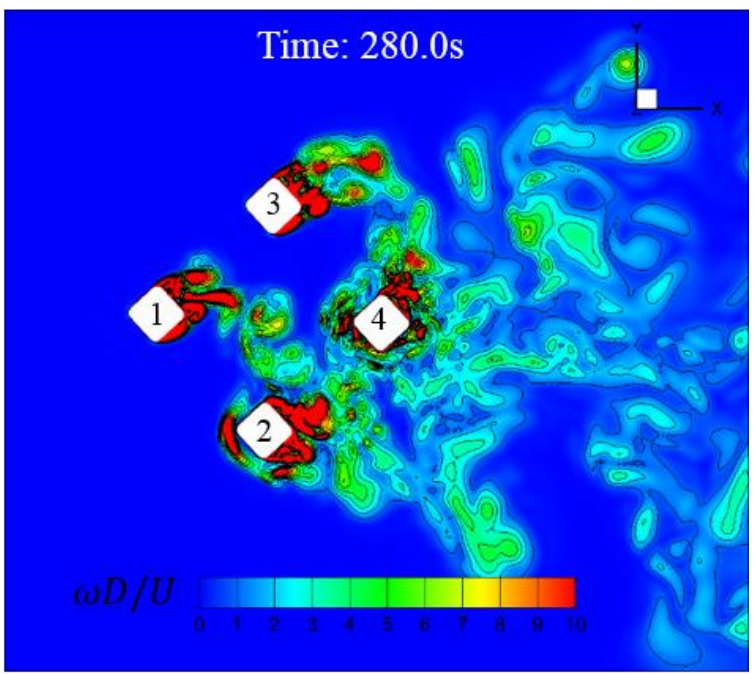

e) "lock-in" $(U r=6.6)$

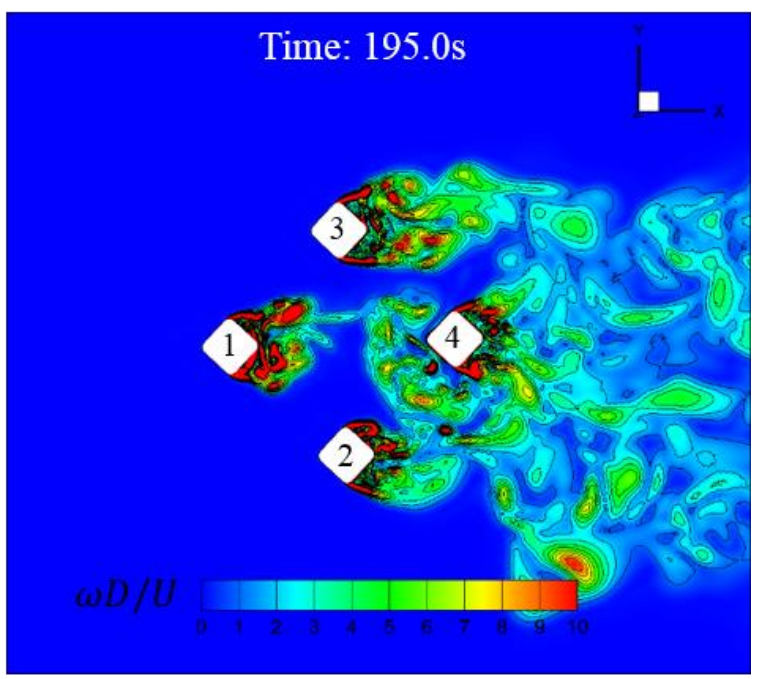

f) "post lock-in" $(U r=12.1)$ 
427 Fig. 20. Non-dimensional vorticity magnitude $(\omega D / U)$ contours of the DDS model for "pre lock-in",

428 "lock-in" and "post lock-in" regions for $0^{\circ}$ and $45^{\circ}$ incidence, $X Y$ plane at middle draft location of the 429 DDS.

430 Fig. 20 presents the three-dimensional vorticity magnitude $\left(\omega D / U\right.$, where $\omega=\sqrt{\left.\left(\omega_{x}^{2}+\omega_{y}^{2}+\omega_{z}^{2}\right)\right)}$. As

431 can be seen, under $45^{\circ}$ incidence, in the "pre lock-in" region, the vortices that form and then detach

432 from column 1 can only impinge directly on column 3 located downstream. However, in the "lock-in"

433 region, it can be clearly seen that the vortices detached from column 1 directly act on column 2 . As

434 the vortex shedding frequency at this reduced velocity is equal to the natural frequency of the

435 structure, the vortices acting on the structure become synchronised with the model oscillation

436 frequency. This is the reason that causes "lock-in" to occur. In the "post lock-in" region, the vortices

437 detached from column 1 strongly act on the column 3 again, and the vortex shedding frequency starts

438 to more away from the natural frequency of the structures resulting in the decreased structure motion.

439 At $0^{\circ}$ incidence, the phenomenon of VIM are similar to that at $45^{\circ}$ incidence. Since the column

440 leading surfaces are vertically faced to the current, the vortices that are detached from the upstream

441 columns are not as significant as in the $45^{\circ}$ incidence cases. However, it can still be clearly seen that

442 in the "lock-in" region, that the vortices detached from the upstream columns directly act on the

443 downstream columns. 

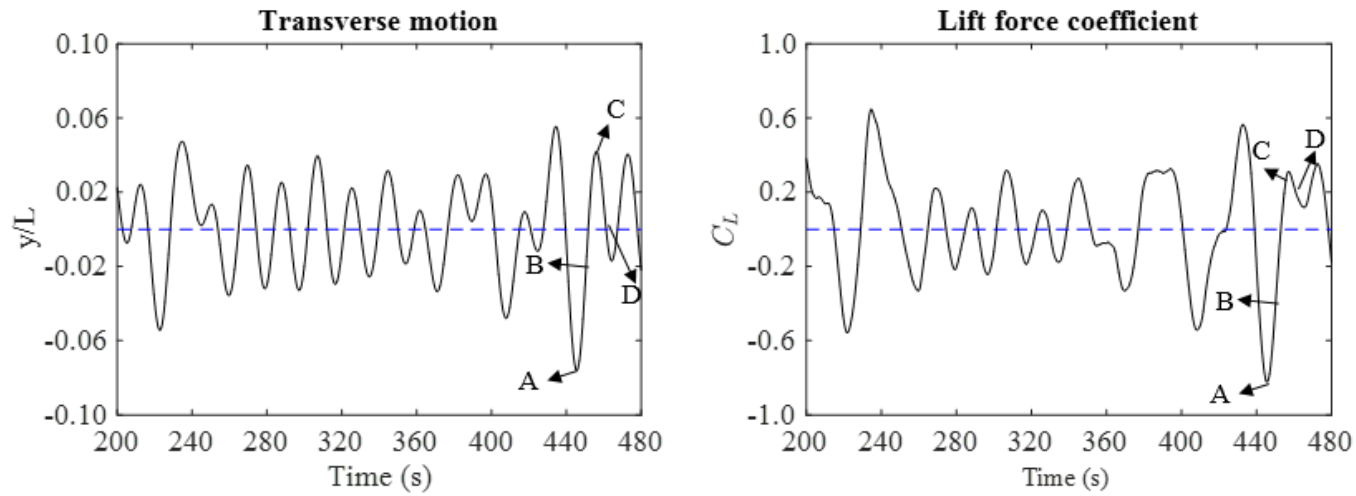

(A)

Time: $445.6 \mathrm{~s}$

(B)

Time: $450.4 \mathrm{~s}$
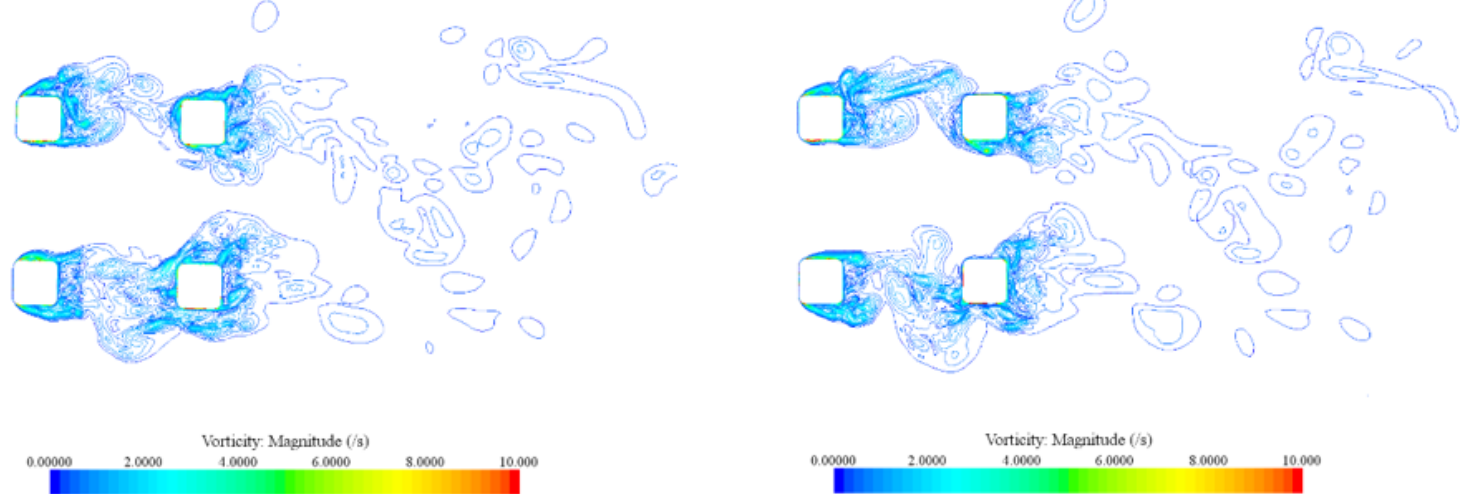

(C)

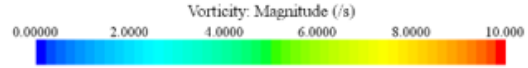

(D)

Time: $460.8 \mathrm{~s}$
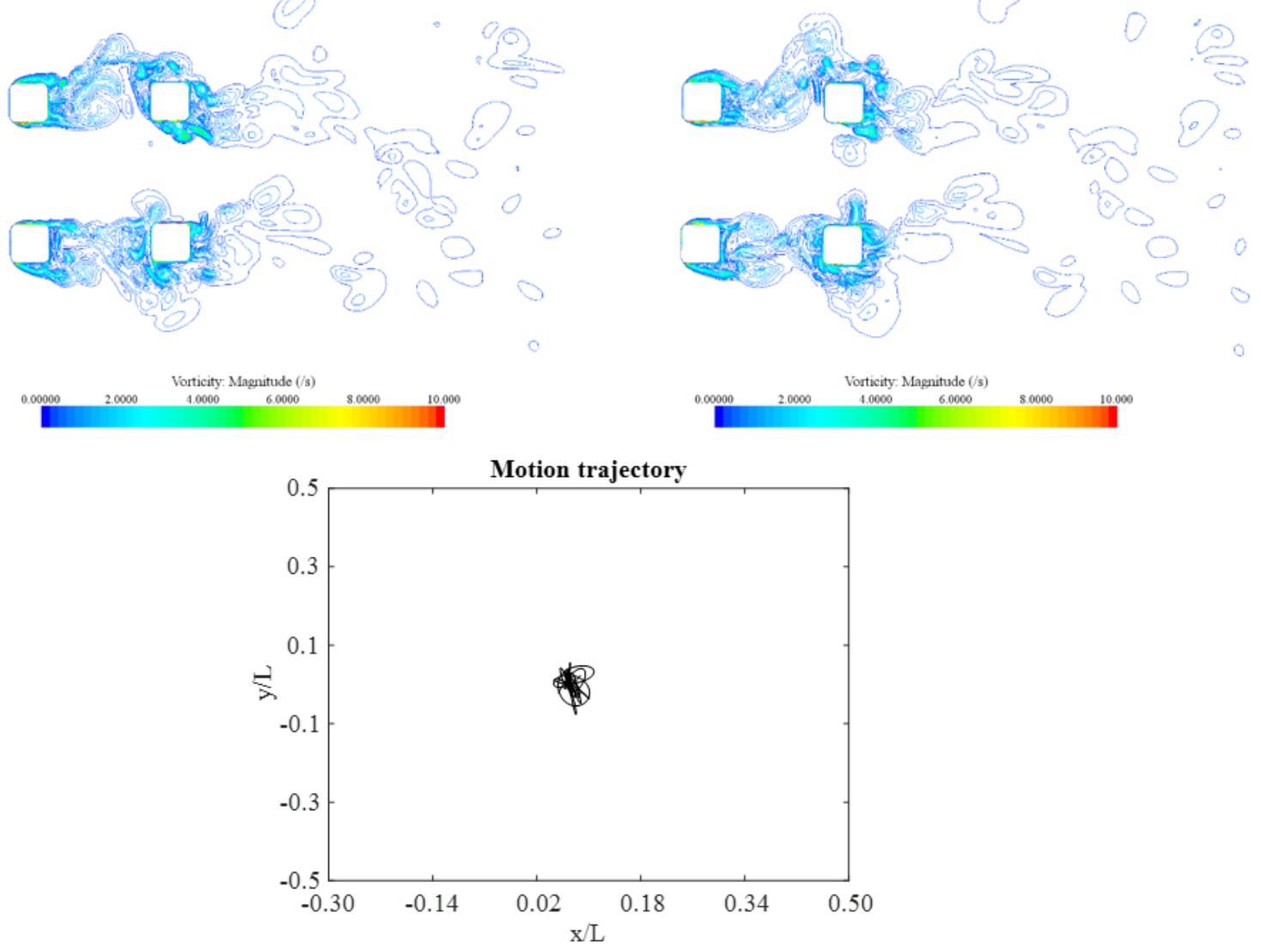
446 Fig. 21. Time history of the motion in the transverse direction $(y / L)$, lift force coefficient $\left(C_{L}\right)$ for $0^{\circ}$ 447 incidence at $U r=3.9$ (pre lock-in), the vorticity contours in the $X Y$ plane at middle draft of the DDS 448 (A and $\mathrm{C}$ refer to the point close to the transverse motion peak value within one oscillation period, $\mathrm{B}$ 449 is the vortex shedding process between A and C, D is the vortex shedding process after C, 450 corresponded simulation time are shown in the figure), and the motion trajectory in the $X Y$ plane. 


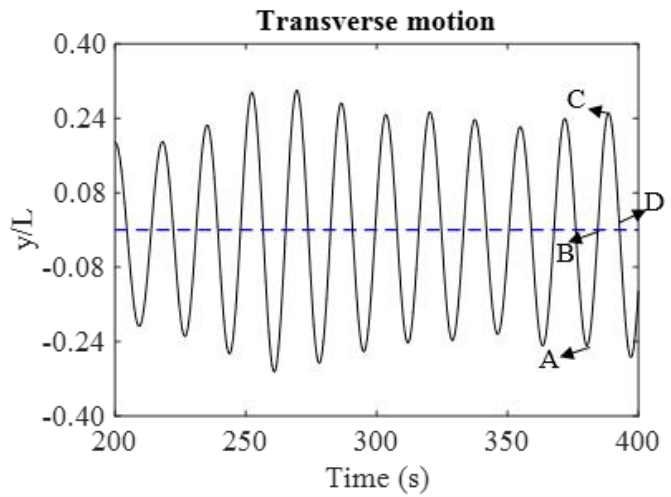

(A)

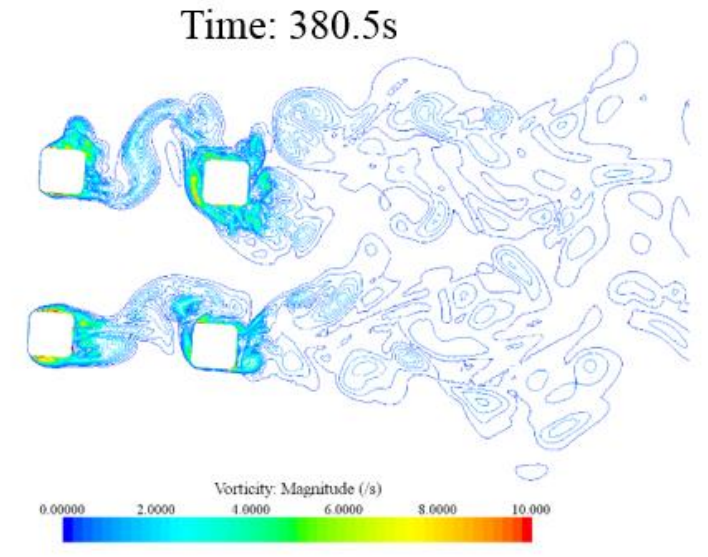

(C)

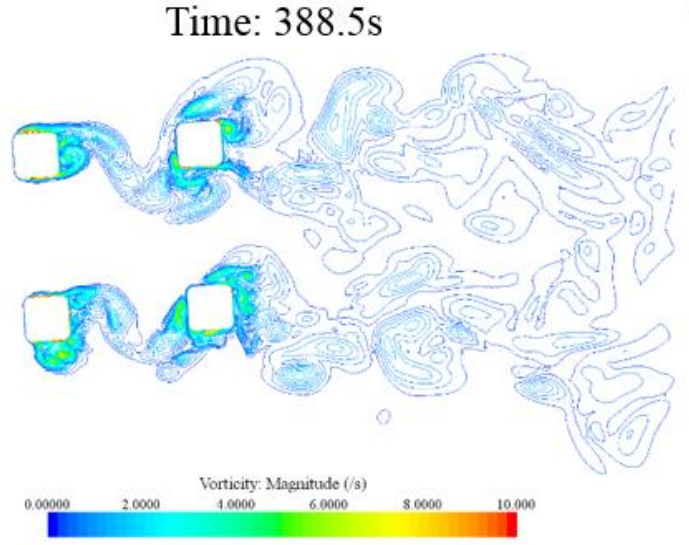

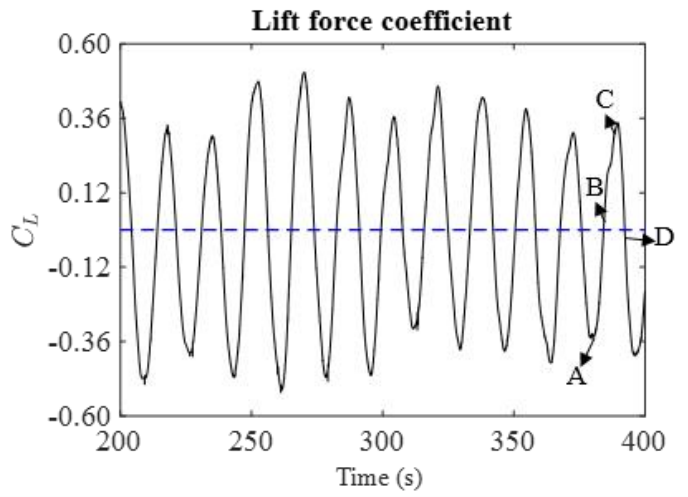

(B)

Time: $384.5 \mathrm{~s}$

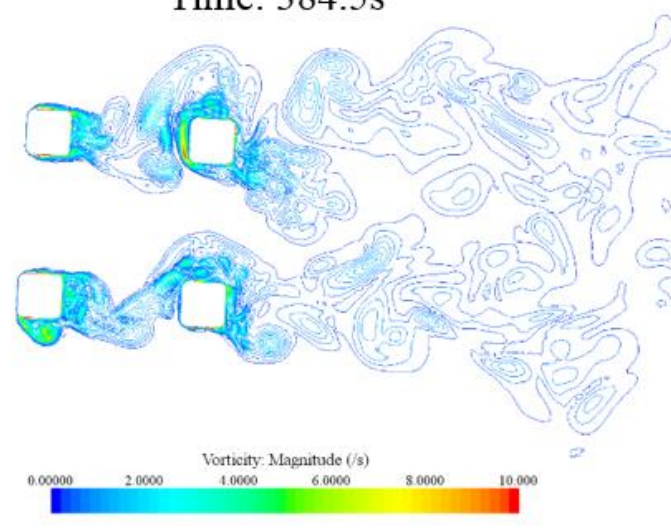

(D)

Time: $392.5 \mathrm{~s}$

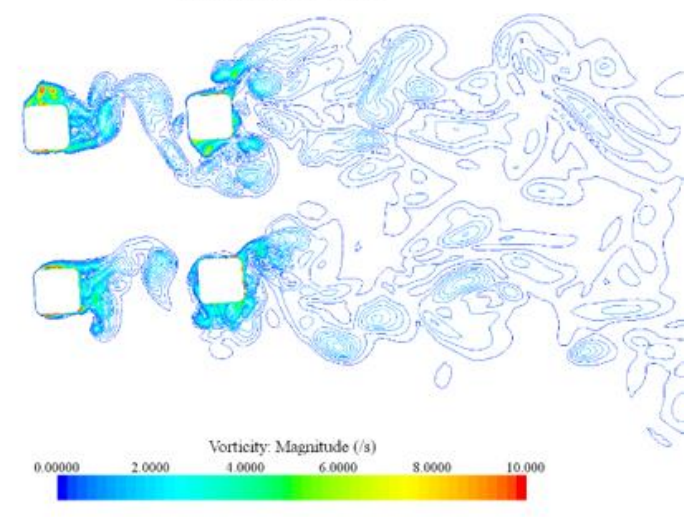

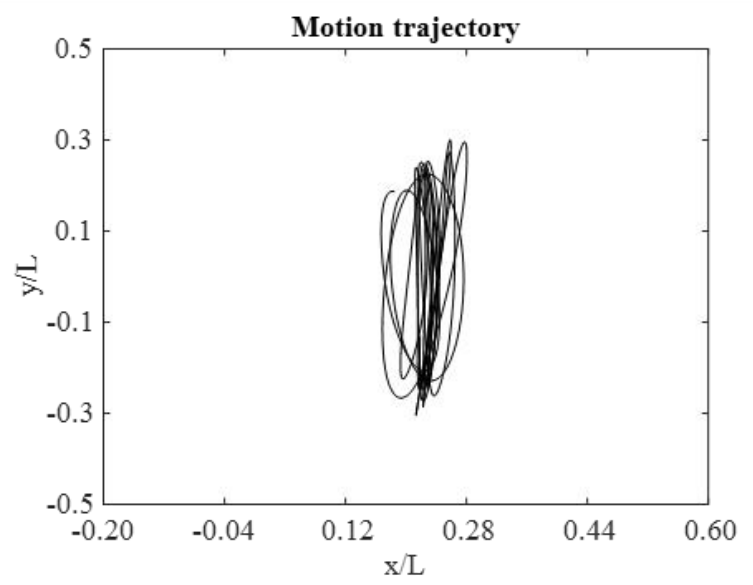


452 Fig. 22. Time history of the motion in the transverse direction $(y / L)$, lift force coefficient $\left(C_{L}\right)$ for $0^{\circ}$ 453 incidence at $U r=6.4$ (lock-in), the vorticity contours in the $X Y$ plane at middle draft of the DDS (A 454 and $\mathrm{C}$ refer to the point close to the transverse motion peak value within one oscillation period, $\mathrm{B}$ is 455 the vortex shedding process between $\mathrm{A}$ and $\mathrm{C}, \mathrm{D}$ is the vortex shedding process after $\mathrm{C}$, corresponded 456 simulation time are shown in the figure), and the motion trajectory in the $X Y$ plane. 

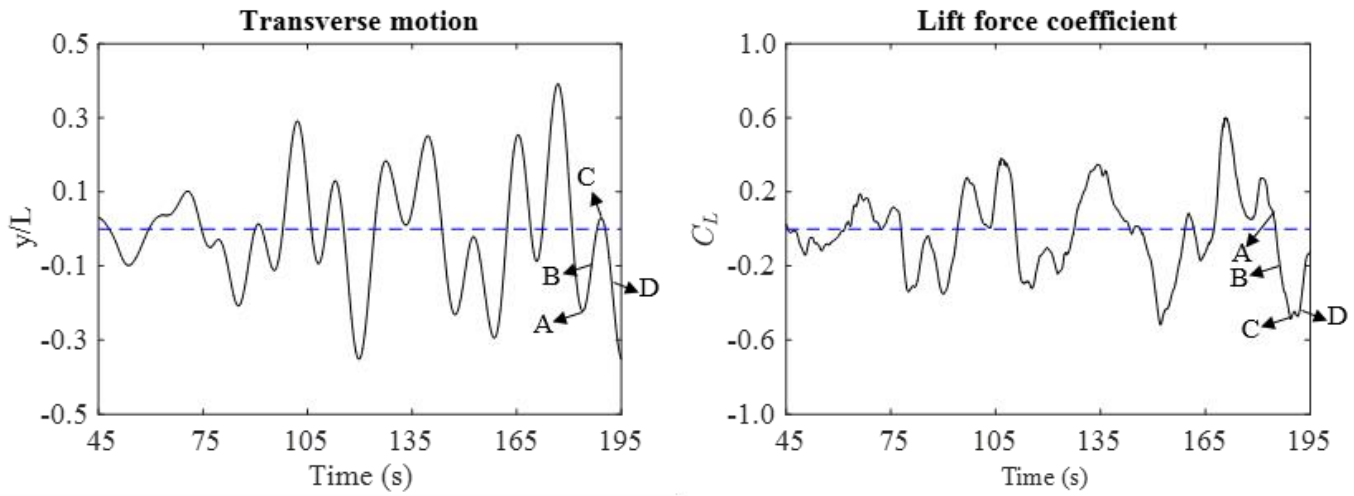

(A)

Time: $183.82 \mathrm{~s}$

(B)

Time: $186.68 \mathrm{~s}$
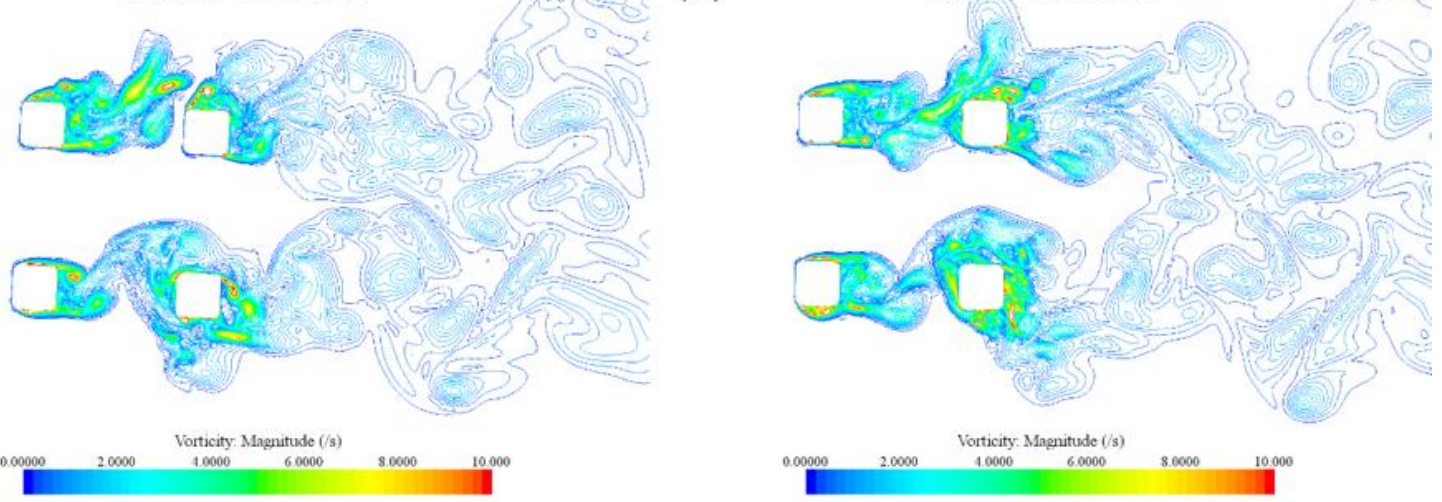

(C)

Time: $189.28 \mathrm{~s}$

(D)

Time: $192.14 \mathrm{~s}$
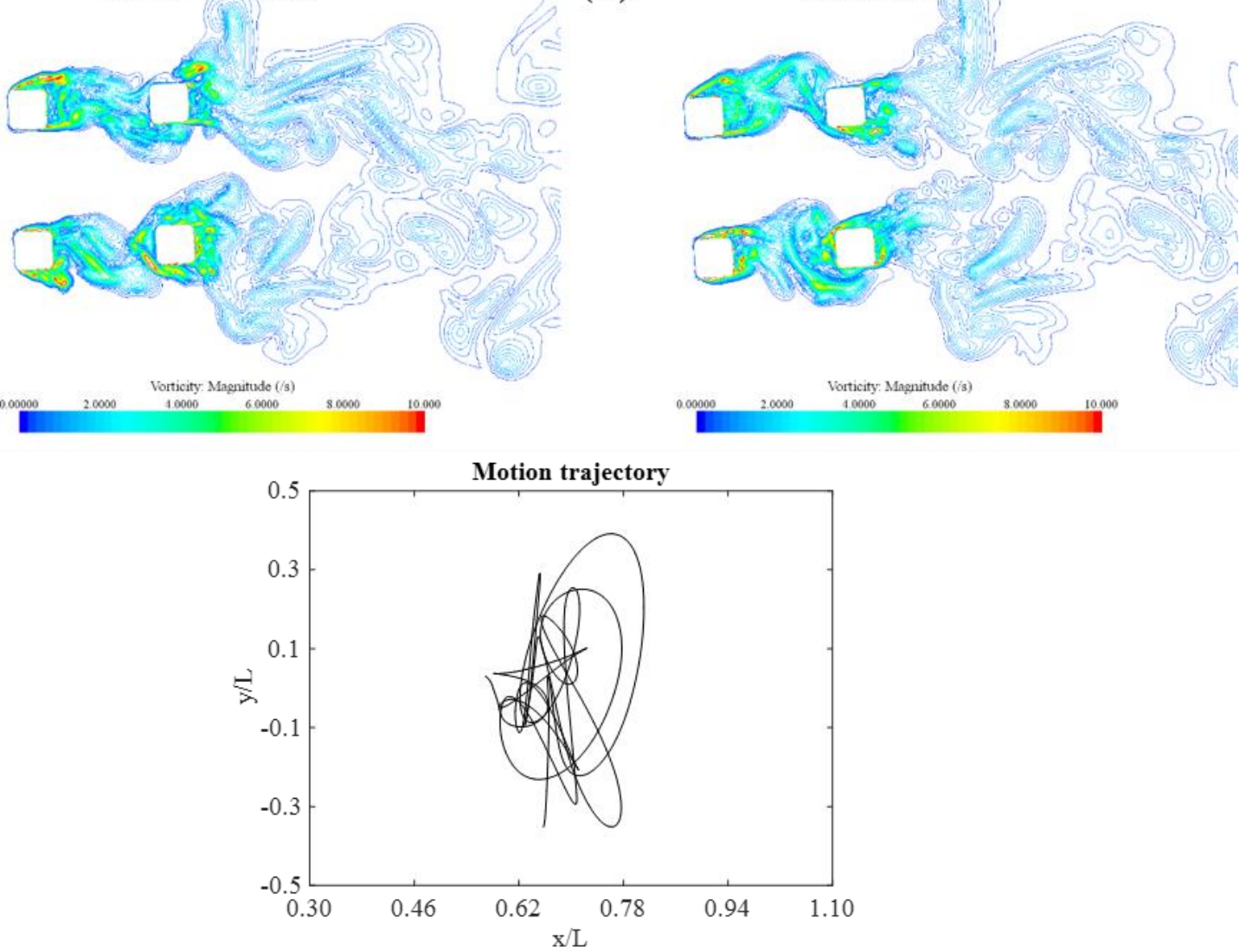

Fig. 23. Time history of the motion in the transverse direction $(y / L)$, lift force coefficient $\left(C_{L}\right)$ for $0^{\circ}$ incidence at $U r=11.8$ (post lock-in), the vorticity contours in the $X Y$ plane at middle draft of the DDS 
460 (A and $\mathrm{C}$ refer to the point close to the transverse motion peak value within one oscillation period, B

461 is the vortex shedding process between $\mathrm{A}$ and $\mathrm{C}, \mathrm{D}$ is the vortex shedding process after $\mathrm{C}$,

462 corresponded simulation time are shown in the figure), and the motion trajectory in the $X Y$ plane. 


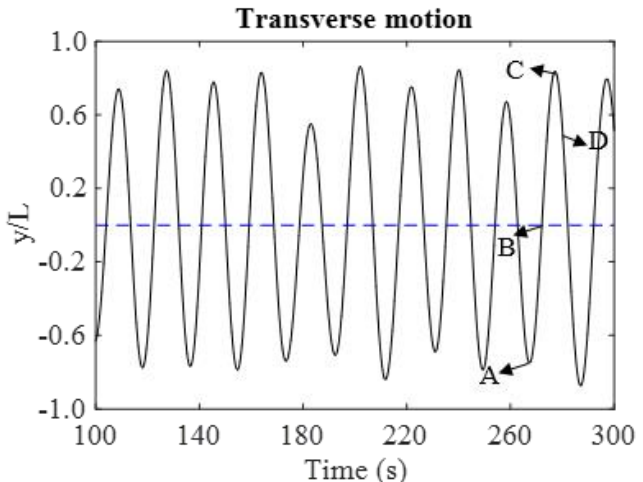

(A)

Time: $267.52 \mathrm{~s}$

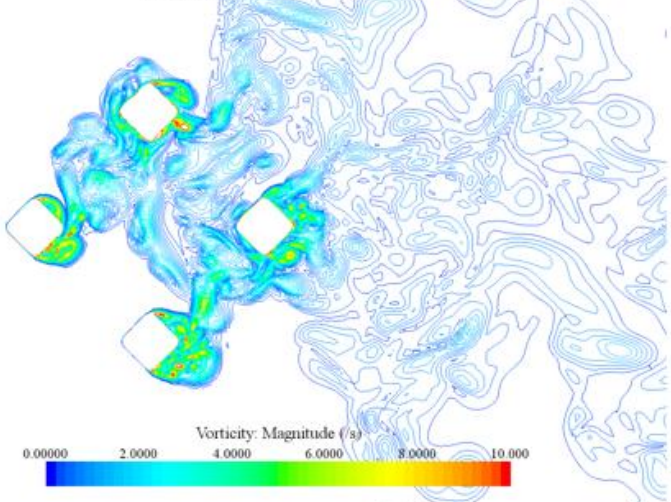

(C)

Time: $277.12 \mathrm{~s}$

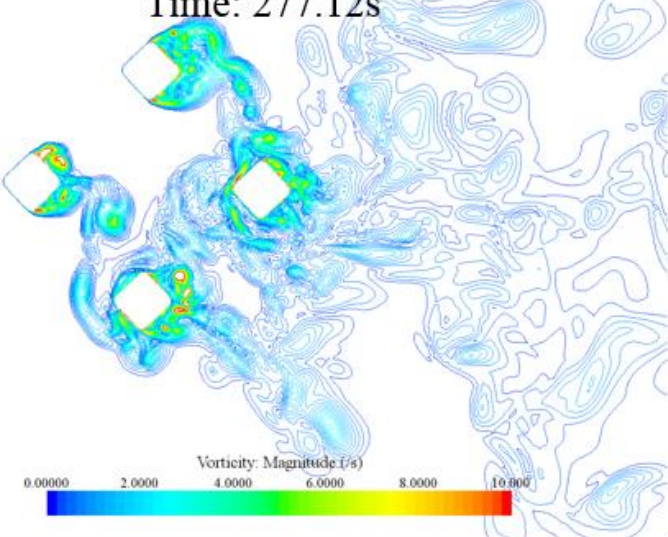

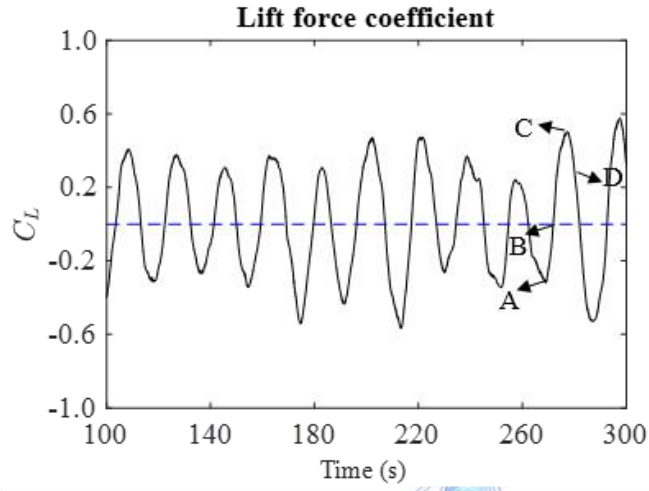

(B)

Time: $272.00 \mathrm{~s}$

(D)

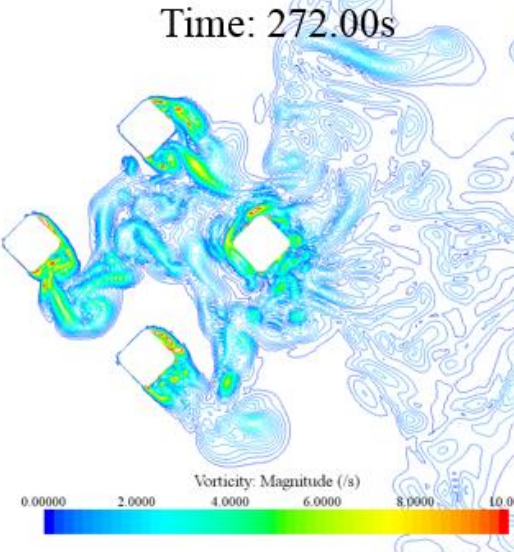

Time: $280.00 \mathrm{~s}$

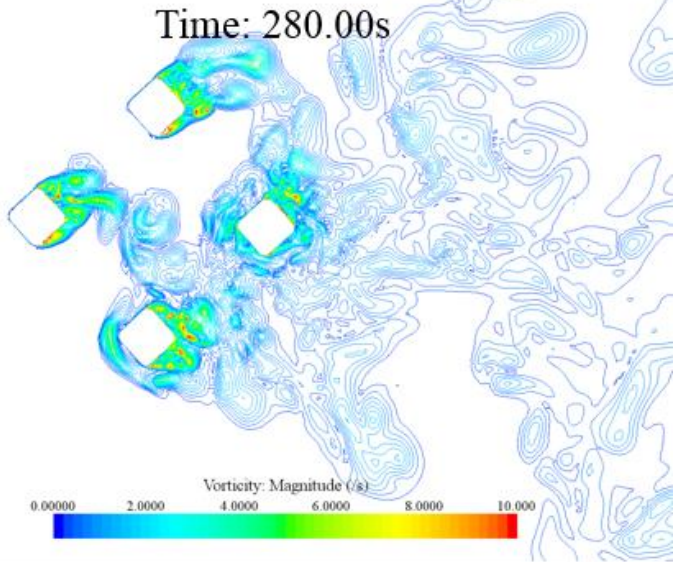

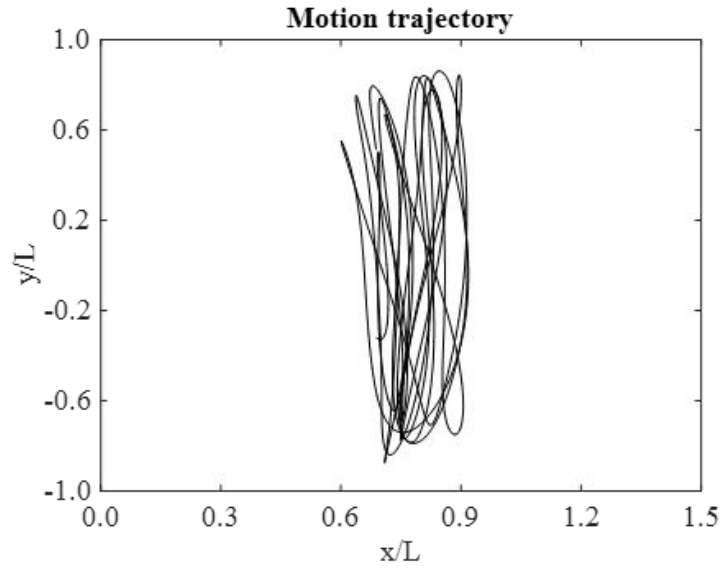

464 Fig. 24. Time history of the motion in the transverse direction $(y / L)$, lift force coefficient $\left(C_{L}\right)$ for $45^{\circ}$ 465 incidence at $U r=6.6$ (lock-in), the vorticity contours in the $X Y$ plane at middle draft of the DDS (A 
and $\mathrm{C}$ refer to the point close to the transverse motion peak value within one oscillation period, $\mathrm{B}$ is the vortex shedding process between $\mathrm{A}$ and $\mathrm{C}, \mathrm{D}$ is the vortex shedding process after $\mathrm{C}$, corresponded simulation time are shown in the figure), and the motion trajectory in the $X Y$ plane.

The flow pattern at the peak transverse motion point may reveal the key factor which induced the VIM. The vortex shedding patterns, which are close to the transverse motion peak value within one oscillation period, are shown in Fig. 21, Fig. 22, Fig. 23 and Fig. 24. Additionally, sub-picture (B) presents the vortex shedding process between the two peak point and sub-picture (D) presents the vortex shedding process after the peak point $\mathrm{C}$ (see Fig. 21, Fig. 22, Fig. 23 and Fig. 24), in order to show the continuous vortex shedding process within one VIM oscillation period.

Fig. 21 presents the time history of the transverse motions, the lift force coefficient the vorticity contours and the motion trajectory under $0^{\circ}$ incidence at $U r=3.9$ ("pre lock-in"). The sub-pictures A, $\mathrm{B}, \mathrm{C}$ and $\mathrm{D}$ show the vorticity contours within one complete vortex shedding period. As can be seen in Fig. 21, the vortices shed from the upstream columns directly impinge on the front faces of the downstream columns. The downstream columns correspondingly break or degrade the vortices being shed from the upstream columns. Unlike the vortices shed from the upstream columns, only relatively small vortices can be seen in the wake region of the downstream columns. Thus the vortices are broken into small elements with weak vortices by the downstream columns. As the small vortices are asymmetrical, generated by breaking the vortices shed from the upstream columns, the corresponding lift force are fluctuating asymmetrically as well. The time histories of the transverse motions and the lift forces show the same trend in Fig. 21.

Similar to Fig. 21, Fig. 22 presents the time history of the transverse motions, the lift force coefficients, the vorticity contours and the motion trajectory under $0^{\circ}$ incidence at the higher reduced velocity of $U r=6.4$ ("lock-in"). With the increase of $U r$, in the "lock-in" region, the vortices shed from the upstream columns act on the downstream columns as if vortices being "shed" of a significant nature from the downstream column itself. The vortices are nearly symmetrically generated on the downstream area of the platform and the vortex street can be clearly seen in the vorticity contours in Fig. 22. This makes both the $C_{L}$ variations and the time history of the transverse motions to become more symmetrical. Because the vortex shedding frequency is close to the overall structure's motion frequency in the transverse direction, the structure's motion trajectory in the transverse direction is nearly same as the vortex shedding trajectory. Hence, the motions of the downstream columns do not break apart the vortices shed from the upstream columns. The upstream formed vortices are acting together with the downstream formed vortices thus to enhance the motions of the structure. Moreover, as the vortices shed from the upstream columns are not effectively broken by the downstream columns, the whole wake region of the structure is significantly enhanced. Similarly, the non- 
dimensional force fluctuations and corresponding motions are induced by the enhanced wake region. This can be clearly seen in Fig. 22. It is also to be noted that the trends of the lift force coefficient and the transverse motions are nearly the same indicating that when the lift force reaches to a peak value, the transverse motion also approaches a peak value.

In the "post lock-in" region, as shown in Fig. 23, the downstream columns break the vortices shed from the upstream columns. Due to the vortex shedding frequency increasing more rapidly than the structure's motion frequency (this can be seen by comparing Fig. 8 and Fig. 10), the vortices shed from the upstream columns are broken by the lateral motion of the downstream columns. However, as the current speed increases, the strength of the vortices is stronger than in the "pre lock-in" region. Although the vortices shed from the upstream columns are seen broken by the downstream columns, "strong vortices" still can be found in the downstream area of the platform. However, the vortex street is not clearly seen as the case in the "lock-in" region. The vortices show a disordered structure in the downstream area. The time history of the lift force coefficient similarly becomes irregular and has less correlation with the time history of the transverse motions. The transverse motion still however has a general trend similarity to the lift force coefficient.

When the flow incidence changes to $45^{\circ}$, the attack angle of the columns makes the transverse motions more pronounced than that for the $0^{\circ}$ incidence condition. With the attack angle of $45^{\circ}$, the columns are not vertically faced to the current. When the vortices shed from the upstream column hit on the downstream column leading faces and edge, the vortex energy explodes and spreads far more on the transverse direction compared with $0^{\circ}$ incidence. Because three columns are on the downstream area at $45^{\circ}$ incidence, the vortex street is more complicated compared with the vortex street at $0^{\circ}$ incidence. Fig. 24 presents the time histories of the lift force coefficient, the motions, the vorticity contours and the motion trajectory at $45^{\circ}$ incidence. It is seen that the trends of the time history of $C_{L}$ and the transverse motions fluctuations are nearly the same. Good correlation between the lift force and the transverse motions is also observed.

The motion trajectories are also plotted in Fig. 21, Fig. 22, Fig. 23 and Fig. 24. According to the $0^{\circ}$ incidence results, there are no eight-shaped trajectories appeared. However, at $45^{\circ}$ incidence, the eight-shaped trajectory can be found in the "lock-in" region as those typically presented for single cylindrical structures.

\section{Conclusions}

This paper presents an experimental and numerical study focusing on various aspects of the VIM of a DDS. While model tests conducted in towing tank served as a reliable benchmark for validating the 
numerical model, it also provided comprehensive measurements on the motion responses and associated forces acting on the structure. Numerical simulation on the other hand, provided substantial details on the vortex shedding characteristics under different current incidence angles and wide range of current strength which further adds to the in-depth analysis of the correlations between the vortex shedding flow characteristics and motion induced.

For two flow incidences investigated, VIM behaviour of the DDS in the horizontal $X Y$ plane occurs in a range of $4.0 \leq U r \leq 11.0$, with peaks around $6.0 \leq U r \leq 7.0$ corresponding to "lock-in". When $U r \geq 15.0$, a high vortex shedding frequency appeared (galloping) is observed. In the "post lock-in" region, the motion response may be dominated by both VIM and galloping. Both in-line and transverse motions under $45^{\circ}$ incidence are larger than that in the $0^{\circ}$ incidence condition with yaw motions showing opposite responses.

Good correlation has been demonstrated among the vortex shedding patterns, the fluctuation forces on the structure, and the VIM trajectory in the present work. The "lock-in" phenomenon was found to have the most striking effect on the vortex shedding processes, the force and the VIM trajectories. During the "lock-in", the vortices shed from the upstream columns of the DDS act on the downstream columns as if vortices being "shed" of a significant nature from the downstream column itself.

It is worth noting that the differences of the mooring line settings between the experiments and numerical simulations may affect the forces on the structures. In order to improve the accuracy of the numerical simulations, a further study considering the gravity force on and the material characteristics of the mooring lines is needed to examine their effects in the numerical model properly.

\section{Acknowledgment}

The authors would like to acknowledge the support of Newton Fund of Royal Academy of Engineering UK (NRCP/1415/211) and the National Natural Science Foundation of China (Grant No. 51279104). This work made use of the facilities of N8 HPC Centre of Excellence, provided and funded by the N8 consortium and EPSRC (Grant No. EP/K000225/1).

\section{References}

[1] Kokkinis T, Sandström RE, Jones HT, Thompson HM, Greiner WL. Development of a Stepped Line Tensioning Solution for Mitigating VIM Effects in Loop Eddy Currents for the Genesis Spar. ASME 2004 23rd International Conference on Offshore Mechanics and Arctic Engineering: American Society of Mechanical Engineers; 2004. p. 995-1004. 
[2] Fujarra ALC, Rosetti GF, de Wilde J, Gonçalves RT. State-of-art on vortex-induced motion: a comprehensive survey after more than one decade of experimental investigation. ASME 2012 31st International Conference on Ocean, Offshore and Arctic Engineering: American Society of Mechanical Engineers; 2012. p. 561-82.

566 [3] Finn LD, Maher JV, Gupta H. The cell spar and vortex induced vibrations. Offshore Technology 567 Conference, OTC2003.

568 [4] van Dijk RRT, Voogt A, Fourchy P, Mirza S. The effect of mooring system and sheared currents on vortex induced motions of truss spars. ASME 2003 22nd International Conference on Offshore Mechanics and Arctic Engineering: American Society of Mechanical Engineers; 2003. p. 285-92. [5] Irani M, Finn L. Model testing for vortex induced motions of spar platforms. ASME 2004 23rd

572 International Conference on Offshore Mechanics and Arctic Engineering: American Society of 573 Mechanical Engineers; 2004. p. 605-10.

574 [6] Halkyard J, Atluri S, Sirnivas S. Truss spar vortex induced motions: benchmarking of CFD and model tests. 25th International Conference on Offshore Mechanics and Arctic Engineering: American Society of Mechanical Engineers; 2006. p. 883-92.

577 [7] Wang Y, Yang J, Peng T, Li X. Model Test Study on Vortex-Induced Motions of a Floating Cylinder. 578 ASME 2009 28th International Conference on Ocean, Offshore and Arctic Engineering: American 579 Society of Mechanical Engineers; 2009. p. 293-301.

580 [8] Wang Y, Yang J, Peng T, Lu H. Strake design and VIM-suppression study of a cell-truss spar. 581 ASME 2010 29th International Conference on Ocean, Offshore and Arctic Engineering: American 582 Society of Mechanical Engineers; 2010. p. 507-13.

583 [9] Halkyard J, Sirnivas S, Holmes S, Constantinides Y, Oakley OH, Thiagarajan K. Benchmarking of 584 truss spar vortex induced motions derived from CFD with experiments. ASME 2005 24th International 585 Conference on Offshore Mechanics and Arctic Engineering: American Society of Mechanical 586 Engineers; 2005. p. 895-902.

587 [10] Oakley OH, Constantinides Y. CFD truss spar hull benchmarking study. ASME 2007 26th 588 International Conference on Offshore Mechanics and Arctic Engineering: American Society of 589 Mechanical Engineers; 2007. p. 703-13.

590 [11] Thiagarajan KP, Constantinides Y, Finn L. CFD analysis of vortex-induced motions of bare and 591 straked cylinders in currents. ASME 2005 24th International Conference on Offshore Mechanics and 592 Arctic Engineering: American Society of Mechanical Engineers; 2005. p. 903-8.

593 [12] Lefevre C, Constantinides Y, Kim JW, Henneke M, Gordon R, Jang H, et al. Guidelines for CFD 594 Simulations of Spar VIM. ASME 2013 32nd International Conference on Ocean, Offshore and Arctic 595 Engineering: American Society of Mechanical Engineers; 2013. p. V007T08A19-VT08A19.

596 [13] Rijken O, Leverette S. Field measurements of vortex induced motions of a deep draft 597 semisubmersible. ASME 2009 28th International Conference on Ocean, Offshore and Arctic 598 Engineering: American Society of Mechanical Engineers; 2009. p. 739-46. 
599

600

601

602

603

604

605

606

607

608

609

610

611

612

613

614

615

616

617

618

619

620

621

622

623

624

625

626

627

628

629

630

631

632

633

634

[14] Waals OJ, Phadke AC, Bultema S. Flow Induced Motions on Multi Column Floaters. ASME 2007 26th International Conference on Offshore Mechanics and Arctic Engineering: American Society of Mechanical Engineers; 2007. p. 669-78.

[15] Hong Y, Choi Y, Lee J, Kim Y. Vortex-induced motion of a deep-draft semi-submersible in current and waves. The Eighteenth International Offshore and Polar Engineering Conference: International Society of Offshore and Polar Engineers; 2008.

[16] Gonçalves RT, Rosetti GF, Fujarra ALC, Oliveira AC. Experimental study on vortex-induced motions of a semi-submersible platform with four square columns, Part I: Effects of current incidence angle and hull appendages. Ocean Engineering. 2012;54:150-69.

[17] Tan JHC, Magee A, Kim JW, Teng YJ, Zukni NA. CFD Simulation for Vortex Induced Motions of a Multi-Column Floating Platform. ASME 2013 32nd International Conference on Ocean, Offshore and Arctic Engineering: American Society of Mechanical Engineers; 2013. p. V007T08A66-VT08A66. [18] Lee S-K, Chien H-P, Gu H. CFD Study of Deep Draft SemiSubmersible VIM. Offshore Technology Conference-Asia: Offshore Technology Conference; 2014.

[19] Tan JHC, Teng YJ, Magee A, Ly BTH, Aramanadka SB. Vortex Induced Motion of TLP With Consideration of Appurtenances. ASME 2014 33rd International Conference on Ocean, Offshore and Arctic Engineering: American Society of Mechanical Engineers; 2014. p. V002T08A25-VT08A25.

[20] Rijken O, Leverette S. Experimental Study into Vortex Induced Motion Response of Semi Submersibles with Square Columns. ASME 2008 27th International Conference on Offshore Mechanics and Arctic Engineering: American Society of Mechanical Engineers; 2008. p. 263-76.

[21] Rijken O, Schuurmans S, Leverette S. Experimental investigations into the influences of SCRs and appurtenances on DeepDraft Semisubmersible Vortex Induced Motion response. ASME 2011 30th International Conference on Ocean, Offshore and Arctic Engineering: American Society of Mechanical Engineers; 2011. p. 269-79.

[22] Tahar A, Finn L. Vortex Induced Motion (VIM) Performance of the Multi Column Floater (MCF)Drilling and Production Unit. ASME 2011 30th International Conference on Ocean, Offshore and Arctic Engineering: American Society of Mechanical Engineers; 2011. p. 755-63.

[23] van Dijk R, Magee A, van Perryman S, van Gebara J. Model test experience on vortex induced vibrations of truss spars. Offshore Technology Conference: Offshore Technology Conference; 2003.

[24] Liu M, Xiao L, Lu H, Xiao X. Experimental study on vortex-induced motions of a semisubmersible with square columns and pontoons at different draft conditions and current incidences. International Journal of Naval Architecture and Ocean Engineering. 2016.

[25] Shur ML, Spalart PR, Strelets MK, Travin AK. A hybrid RANS-LES approach with delayed-DES and wall-modelled LES capabilities. International Journal of Heat and Fluid Flow. 2008;29:1638-49. [26] Spalart PR, Jou WH, Strelets M, Allmaras SR. Comments on the feasibility of LES for wings, and on a hybrid RANS/LES approach. Advances in DNS/LES. 1997;1:4-8. 
635 [27] Spalart PR, Deck S, Shur ML, Squires KD, Strelets MK, Travin A. A new version of detached636 eddy simulation, resistant to ambiguous grid densities. Theoretical and Computational Fluid Dynamics. $637 \quad 2006 ; 20: 181-95$.

638 [28] CD-adapco. User Guide. Star-CCM+ Version 9.04; 2014.

639 [29] Liu M, Xiao L, Lyu H, Tao L. Numerical Analysis of Pontoon Effect on Flow-Induced Forces of 640 the Deep Draft Semisubmersible in a Cross-Flow. ASME 2015 34th International Conference on Ocean, 641 Offshore and Arctic Engineering: American Society of Mechanical Engineers; 2015. p. V001T01A30642 VT01A30.

643 [30] Schewe G. On the force fluctuations acting on a circular cylinder in crossflow from subcritical up 644 to transcritical Reynolds numbers. Journal of Fluid Mechanics. 1983;133:265-85.

645 [31] Sarpkaya T. A critical review of the intrinsic nature of vortex-induced vibrations. Journal of Fluids 646 and Structures. 2004;19:389-447.

647 [32] Sumer BM, Fredsøe J. Hydrodynamics around cylindrical structures: World Scientific; 1997. 


\section{List of tables}

650 Table 1. Summary of the studies on VIM of deep-draft structures (“*” is the numerical result).

651 Table 2. Main characteristics of the DDS unit.

652 Table 3. Natural periods of the motions in calm water.

653 Table 4. The main characteristics of the MARIN DDS.

654 Table 5. Numerical set-up information.

655 Table 6. The mesh refinement tests.

656 Table 7. The time step sensitivity study.

657 Table 8. Comparison of $\bar{C}_{D}$ from the present numerical calculation and the MARIN experimental 658 measurements.

659 Table 9. Comparison of results from the present numerical calculations and experimental measurements 660 for $45^{\circ}$ incidence.

661 Table 10. Comparison of the mooring line mean forces for $0^{\circ}$ incidence at $U r=3.9,6.4$ (The mooring 662 lines arrangement is shown in Fig. 3). 


\section{List of figures}

665 Fig. 1. Characteristic dimensions of a DDS.

666 Fig. 2. Experimental set-up in the towing tank.

667 Fig. 3. Schematic of the experimental set-up.

668 Fig. 4. Computational domain.

669 Fig. 5. Visualization of the mesh at the middle draft level of the DDS (XY plane at the middle draft of 670 the DDS).

671 Fig. 6. Convergence line for both $\bar{C}_{D}$ and St.

672 Fig. 7. Non-dimensional in-line and transverse characteristics amplitudes $\left(A_{x} / L, A_{y} / L\right)$, the $U r$ is defined 673 based on $T_{\text {otransverse }}$ ).

674 Fig. 8. FFT of the motions in the transverse direction as a function of $U r$ and $f_{y}$ for $0^{\circ}$ incidence (the $U r$ 675 is defined based on $\left.T_{\text {Otransverse }}\right)$.

676 Fig. 9. FFT of the motions in the transverse direction as a function of $U r$ and $f_{y}$ for $45^{\circ}$ incidence (the $677 U r$ is defined based on $\left.T_{\text {Otransverse }}\right)$.

678 Fig. 10. FFT of lift force coefficient as a function of $U r$ and $f_{s}$ for $0^{\circ}$ incidence (the $U r$ is defined based 679 on $\left.T_{\text {Otransverse }}\right)$.

680 Fig. 11. FFT of lift force coefficient as a function of $U r$ and $f_{s}$ for $45^{\circ}$ incidence (the $U r$ is defined based 681 on $\left.T_{\text {Otransverse }}\right)$.

682 Fig. 12. FFT of the transverse motions and the lift force coefficients at $U r=6.4$ for $0^{\circ}$ incidence, (a) 683 transverse motion $(y / L)$; (b) lift force coefficient $\left(C_{L}\right)$.

684 Fig. 13. FFT of the transverse motions and the lift force coefficients at $U r=6.6$ for $45^{\circ}$ incidence, (a) transverse motion $(y / L)$; (b) lift force coefficient $\left(C_{L}\right)$. 
Fig. 14. FFT of the transverse motions and the lift force coefficients at $U r=15.7$ for $0^{\circ}$ incidence, (a) transverse motion $(y / L)$; (b) lift force coefficient $\left(C_{L}\right)$.

Fig. 15. Significant values of the transverse peaks $\left(A_{1 / 3} / L\right)$. The $U r$ is defined based on $T_{0 \text { transverse }}$.

Fig. 16. Non-dimensional yaw characteristics amplitude (the $U r$ is defined based on $T_{\text {Oyaw }}$ ).

690

Fig. 17. FFT of the yaw motion as a function of $U r$ and $f_{\text {yaw }}$ for $0^{\circ}$ incidence (the $U r$ is defined based on $\left.T_{\text {Oyaw }}\right)$.

Fig. 18. FFT of the yaw motion as a function of $U r$ and $f_{\text {yaw }}$ for $45^{\circ}$ incidence (the $U r$ is defined based 693 on $\left.T_{\text {oyaw }}\right)$.

Fig. 19. Mean drag coefficient $\left(\bar{C}_{D}\right)$, where $A$ is the projected area at $0^{\circ}$ incidence.

Fig. 20. Non-dimensional vorticity magnitude $(\omega D / U)$ contours of the DDS model for "pre lock-in", "lock-in" and "post lock-in" regions for $0^{\circ}$ and $45^{\circ}$ incidence, $X Y$ plane at middle draft location of the DDS.

Fig. 21. Time history of the motion in the transverse direction $(y / L)$, lift force coefficient $\left(C_{L}\right)$ for $0^{\circ}$ incidence at $U r=3.9$ (pre lock-in), the vorticity contours in the $X Y$ plane at middle draft of the DDS (A and $\mathrm{C}$ refer to the point close to the transverse motion peak value within one oscillation period, $\mathrm{B}$ is the vortex shedding process between $\mathrm{A}$ and $\mathrm{C}, \mathrm{D}$ is the vortex shedding process after $\mathrm{C}$, corresponded simulation time are shown in the figure), and the motion trajectory in the $X Y$ plane.

Fig. 22. Time history of the motion in the transverse direction $(y / L)$, lift force coefficient $\left(C_{L}\right)$ for $0^{\circ}$ incidence at $U r=6.4$ (lock-in), the vorticity contours in the $X Y$ plane at middle draft of the DDS (A and $\mathrm{C}$ refer to the point close to the transverse motion peak value within one oscillation period, $\mathrm{B}$ is the vortex shedding process between $\mathrm{A}$ and $\mathrm{C}, \mathrm{D}$ is the vortex shedding process after $\mathrm{C}$, corresponded simulation time are shown in the figure), and the motion trajectory in the $X Y$ plane.

Fig. 23. Time history of the motion in the transverse direction $(y / L)$, lift force coefficient $\left(C_{L}\right)$ for $0^{\circ}$ incidence at $U r=11.8$ (post lock-in), the vorticity contours in the $X Y$ plane at middle draft of the DDS (A and $\mathrm{C}$ refer to the point close to the transverse motion peak value within one oscillation period, $\mathrm{B}$ is the vortex shedding process between $\mathrm{A}$ and $\mathrm{C}, \mathrm{D}$ is the vortex shedding process after $\mathrm{C}$, corresponded simulation time are shown in the figure), and the motion trajectory in the $X Y$ plane. 
713 Fig. 24. Time history of the motion in the transverse direction $(y / L)$, lift force coefficient $\left(C_{L}\right)$ for $45^{\circ}$ 714 incidence at $U r=6.6$ (lock-in), the vorticity contours in the $X Y$ plane at middle draft of the DDS (A and $715 \mathrm{C}$ refer to the point close to the transverse motion peak value within one oscillation period, $\mathrm{B}$ is the 716 vortex shedding process between $\mathrm{A}$ and $\mathrm{C}, \mathrm{D}$ is the vortex shedding process after $\mathrm{C}$, corresponded 717 simulation time are shown in the figure), and the motion trajectory in the $X Y$ plane. 